Supporting Information for

\title{
Visible-Transparent Aromatic Polymers Obtained by the Polycondensation of
}

\section{a Bis(trifluorovinyl)benzene with Bisphenols}

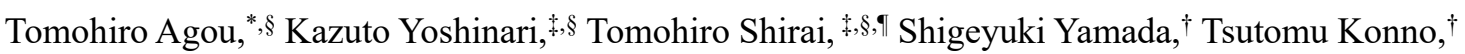
Yoshiyuki Mizuhata, ${ }^{\#}$ Norihiro Tokitoh, ${ }^{\#}$ Sana Yasuda, Takashi Yamazaki, Hiroki Fukumoto, ${ }^{*} \S$ and Toshio Kubota*, ${ }^{*}$

${ }^{\S}$ Department of Quantum Beam Science, Graduate School of Science and Engineering, Ibaraki University, 4-12-1 Nakanarusawa, Hitachi, Ibaraki 316-8511, Japan

"Functional Materials Group, Research and Development Department, TOSOH FINECHEM CORPORATION, 4988 Kaisei-cho, Shunan, Yamaguchi 746-0006, Japan

${ }^{\dagger}$ Faculty of Molecular Chemistry and Engineering, Kyoto Institute of Technology, Matsugasaki, Sakyo-ku, Kyoto, 606-8585, Japan

\# Institute for Chemical Research, Kyoto University, Gokasho, Uji, Kyoto 611-0011, Japan

' Division of Applied Chemistry, Institute of Engineering, Tokyo University of Agriculture and Technology, 2-24-16 Naka-cho, Koganei-shi, Tokyo 184-8588, Japan

$\$$ Equal contributions. 


\section{Table of Contents}

NMR Spectra, HRMS (for $\mathbf{1}$ and $\mathbf{2}$ ) and TG and DSC data (for polymers $\mathbf{3}$ )

$\mathrm{UV} / \mathrm{vis}$ and PL spectra of the polymers $\mathrm{S} 30-\mathrm{S} 32$

Contact angle measurement .S33

Complete citation for ref. 38 S34 
NMR Spectra of the New Compounds

1

${ }^{1} \mathrm{H} \mathrm{NMR}\left(\mathrm{CDCl}_{3}\right)$

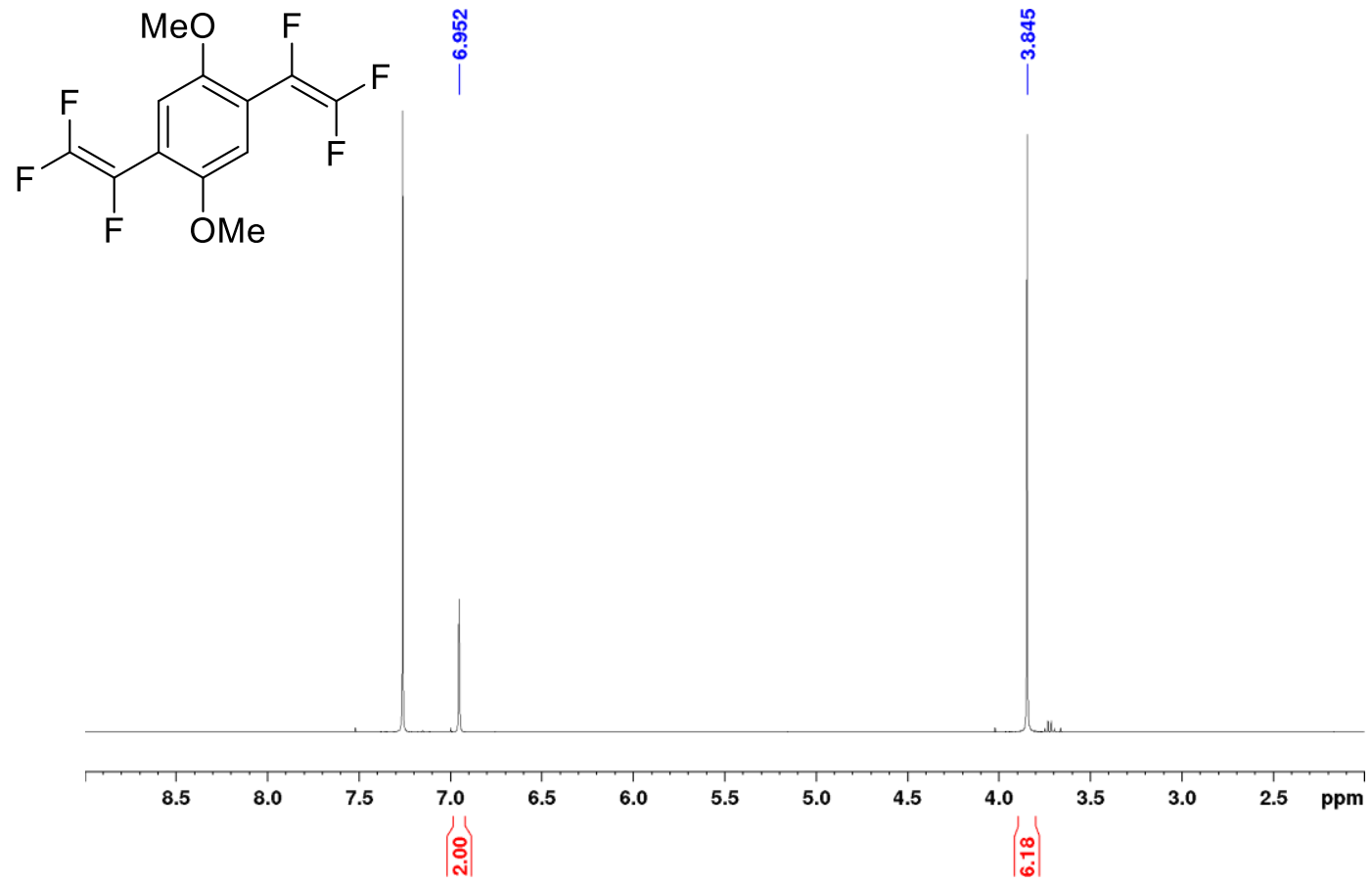

${ }^{19} \mathrm{~F}$ NMR $\left(\mathrm{CDCl}_{3}\right)$

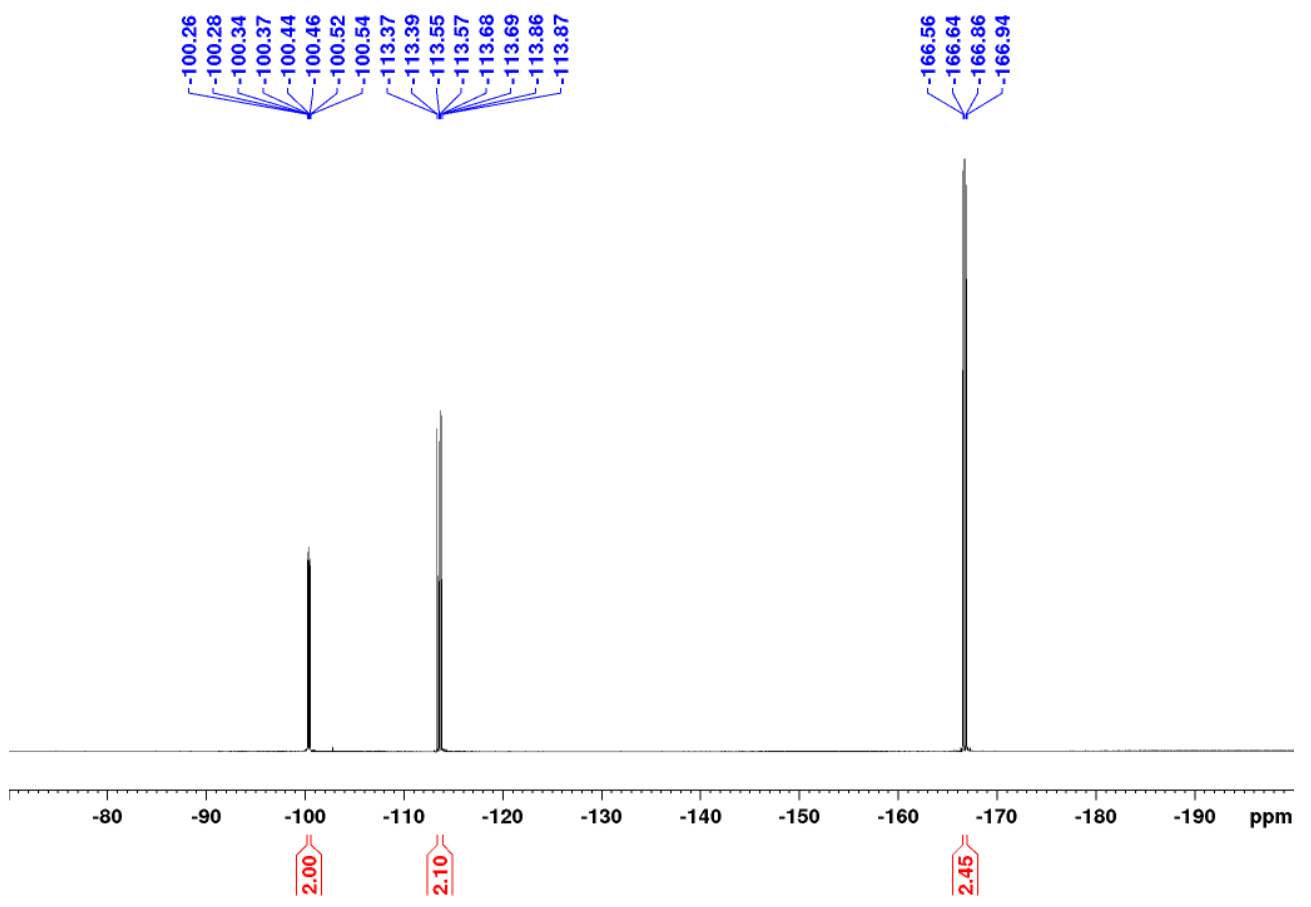


${ }^{13} \mathrm{C} \mathrm{NMR}\left(\mathrm{CDCl}_{3}\right)$

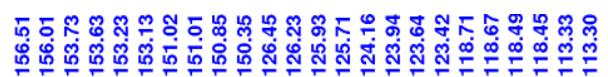

促

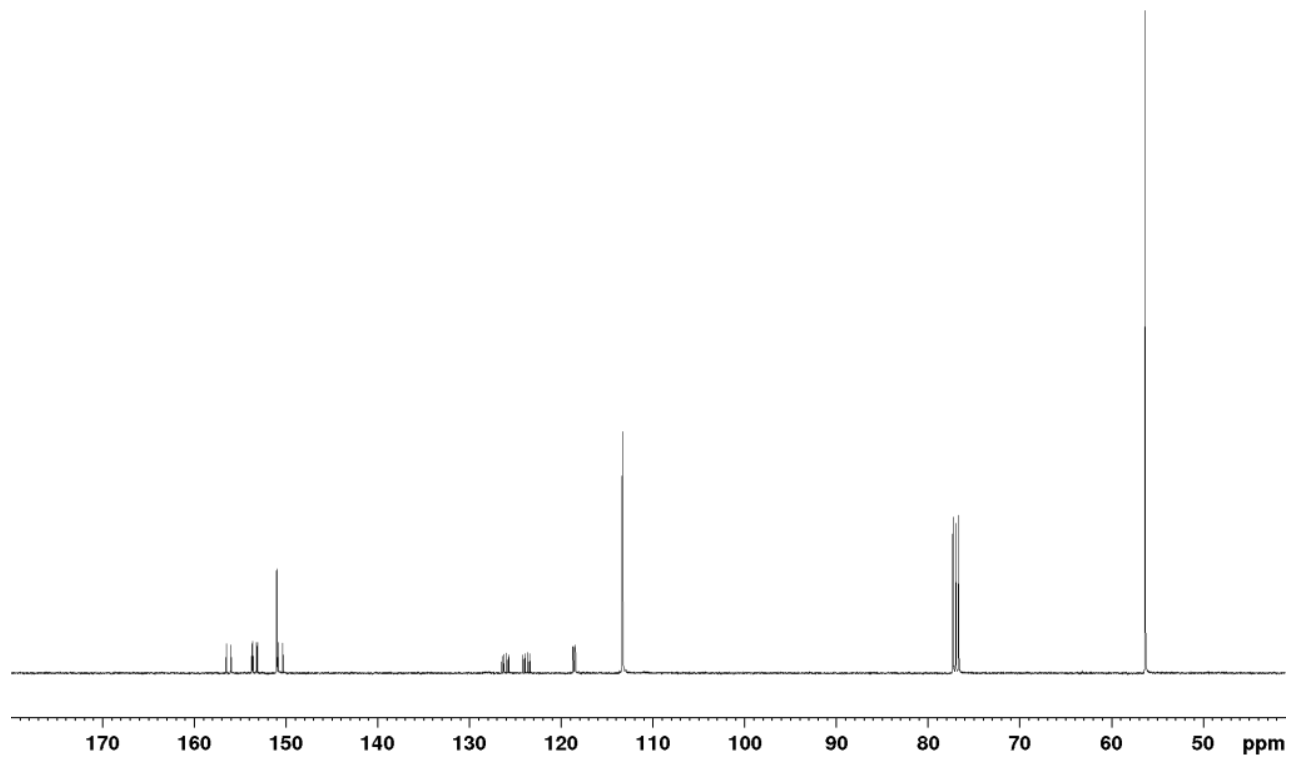




\section{HRMS (APPI+)}

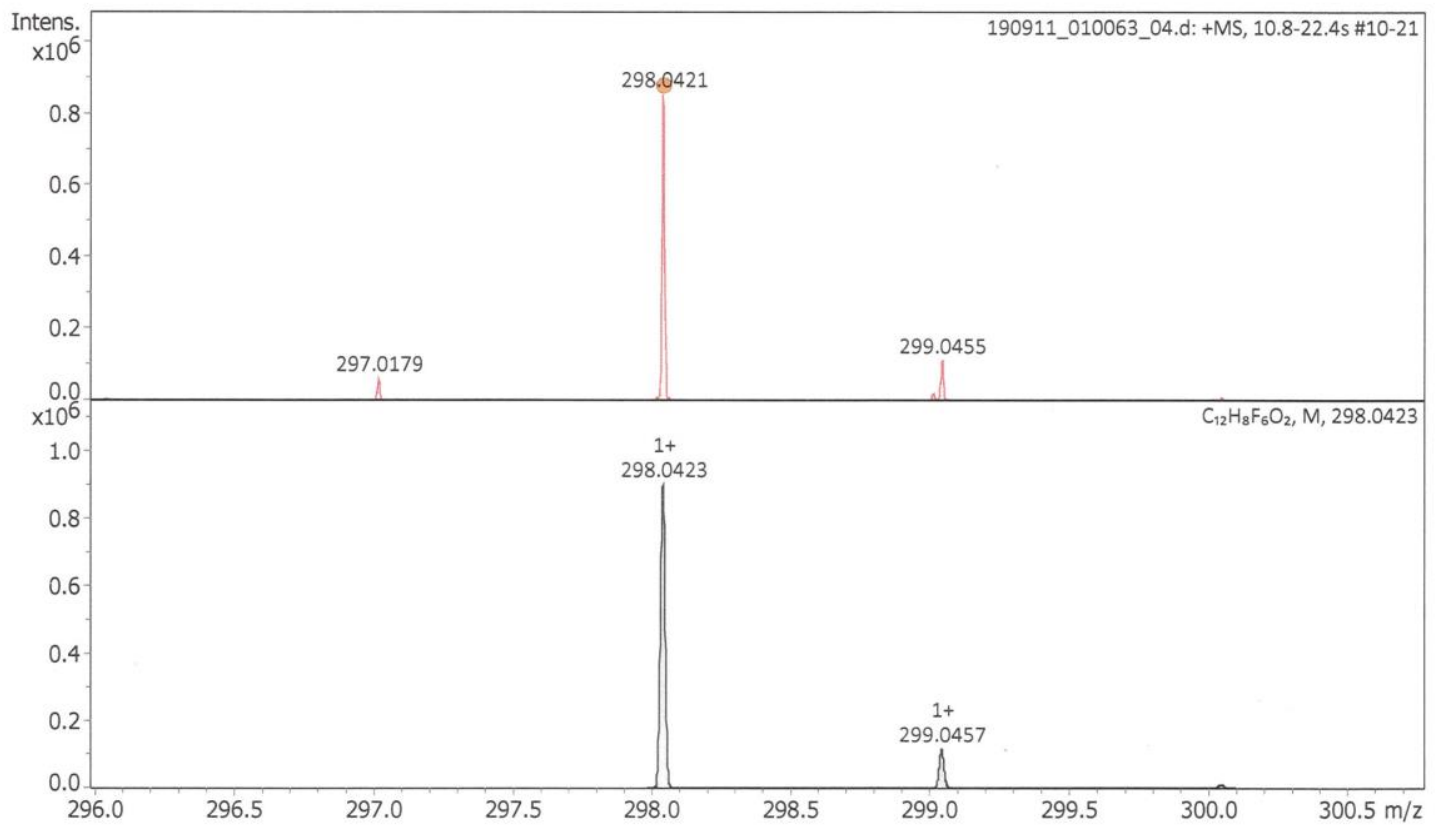

Red: experiment, black: calcd. 
NMR spectrum of the mixture of $\mathbf{2}$ and $\mathbf{2},\left(\mathrm{CDCl}_{3}\right)$

${ }^{1} \mathrm{H} \mathrm{NMR}\left(\mathrm{CDCl}_{3}\right)$

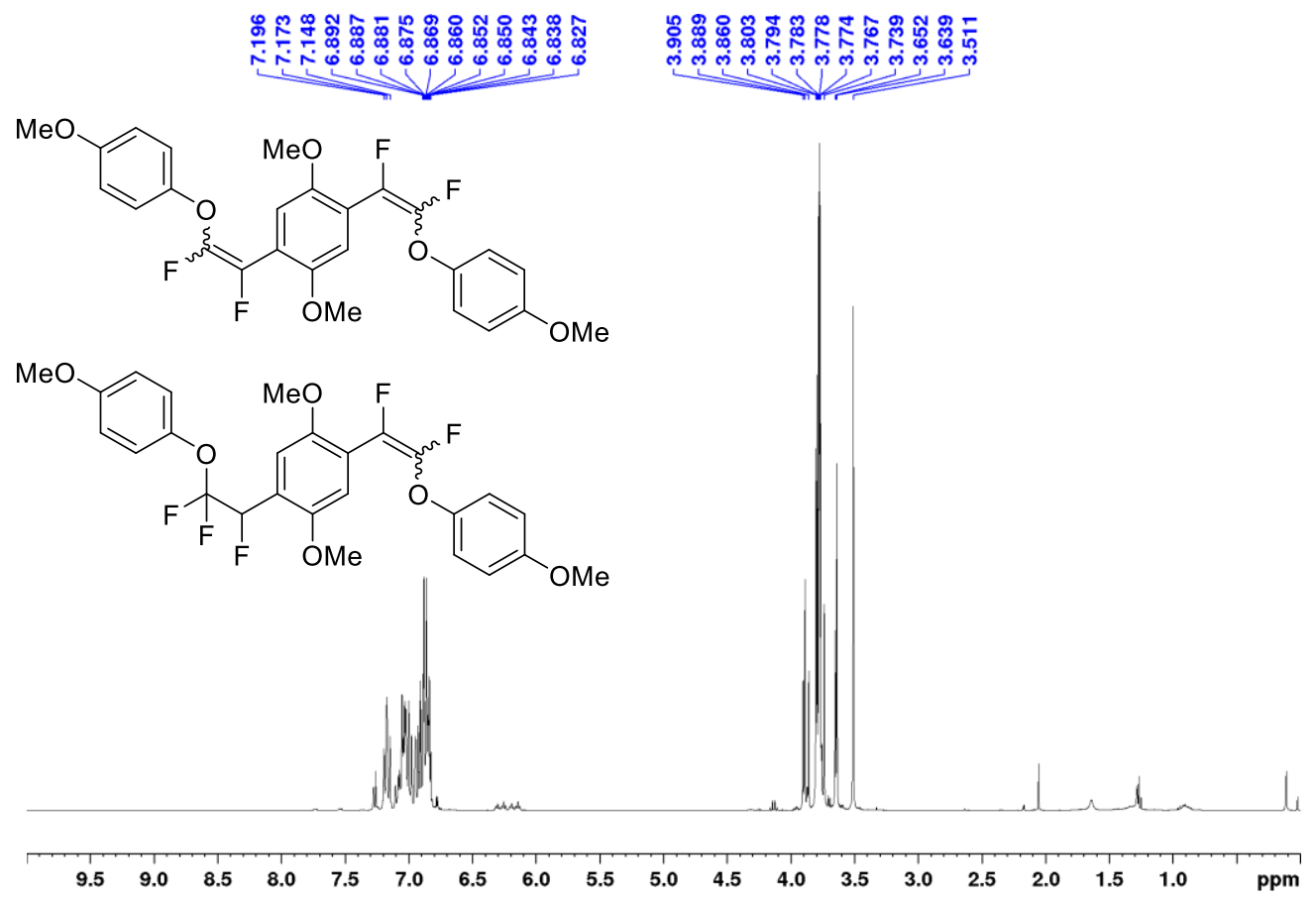

${ }^{19} \mathrm{~F} \mathrm{NMR}\left(\mathrm{CDCl}_{3}\right)$

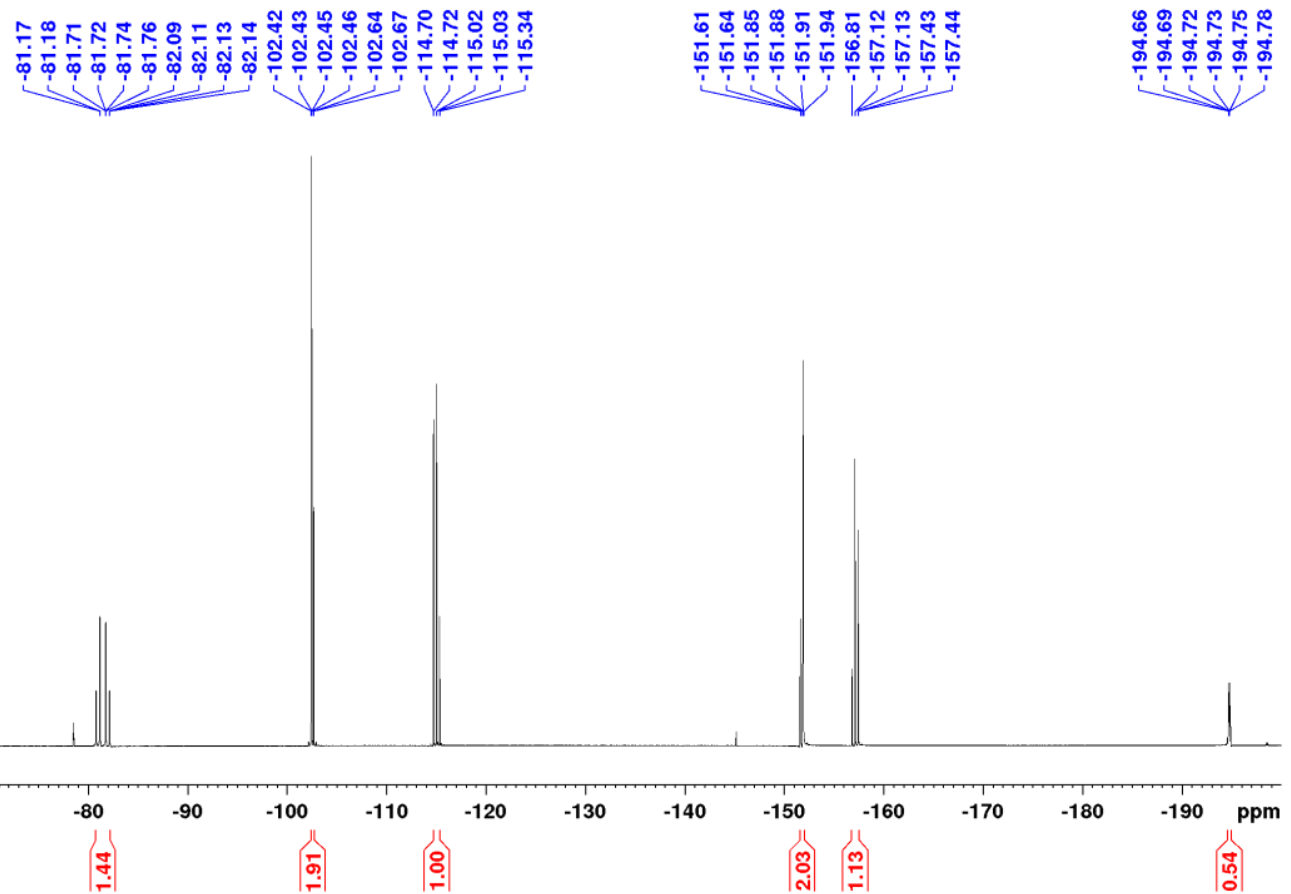


${ }^{13} \mathrm{C} \mathrm{NMR}\left(\mathrm{CDCl}_{3}\right)$

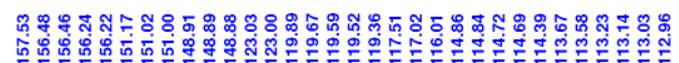

VW

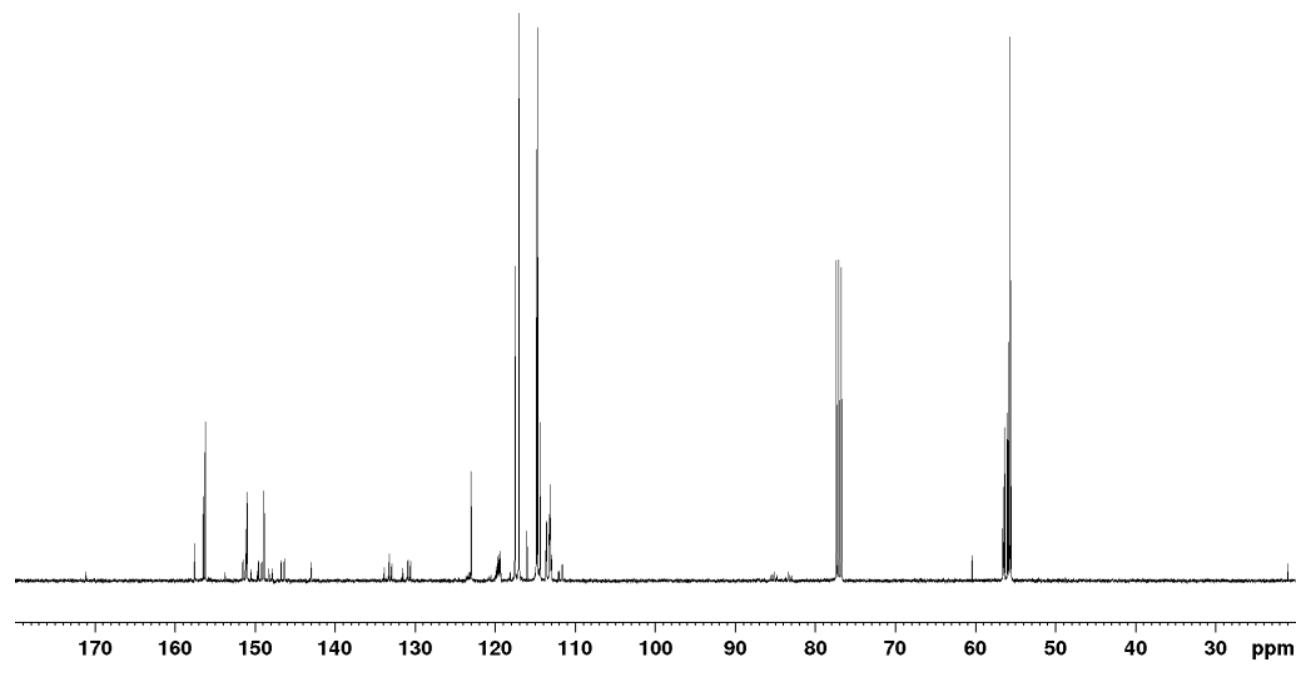




\section{HRMS (APPI+)}

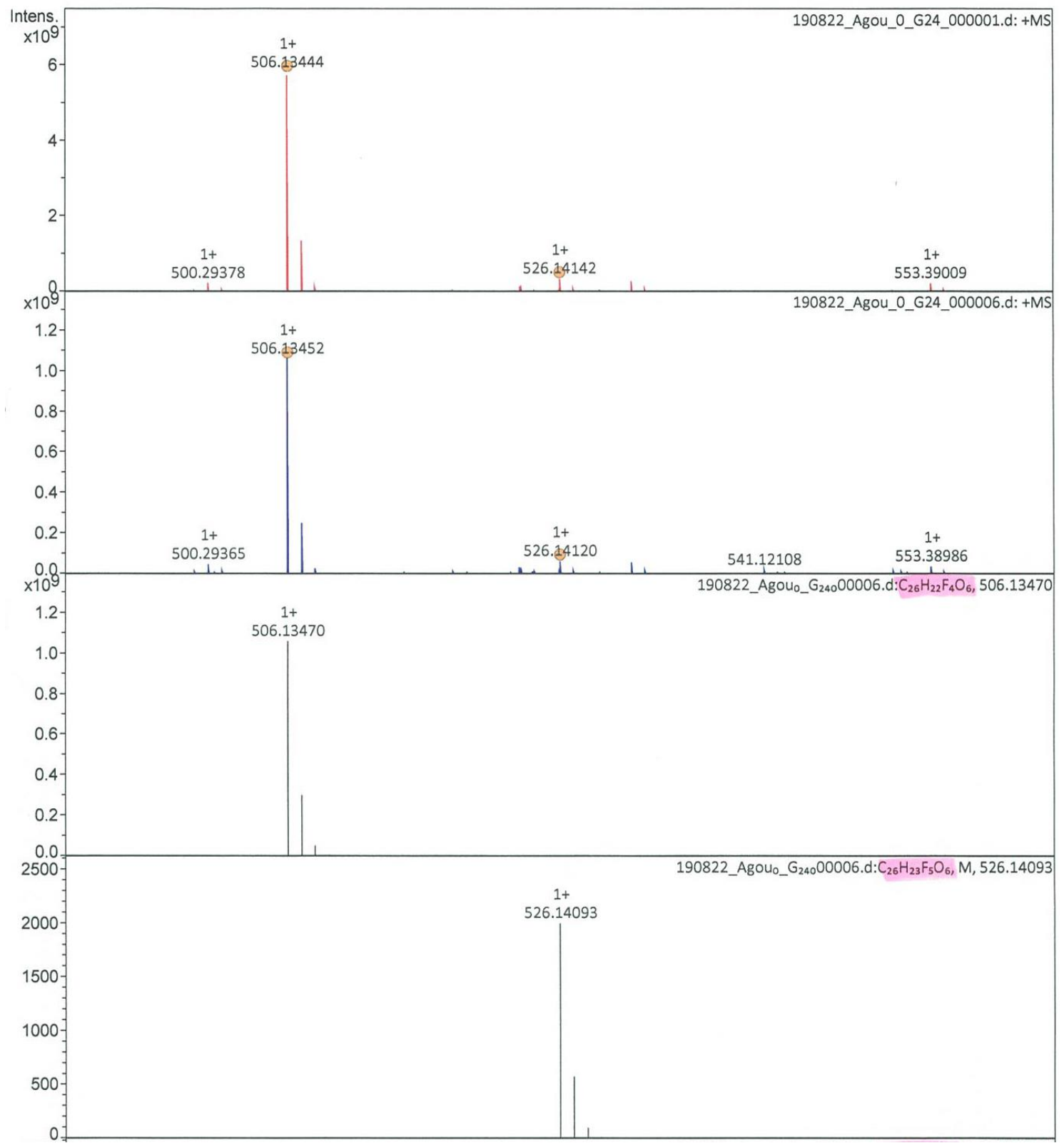

Red: experiment 1, blue: experiment 2, black: calcd. 
3a

${ }^{1} \mathrm{H} \mathrm{NMR}\left(\mathrm{CDCl}_{3}\right)$<smiles></smiles>

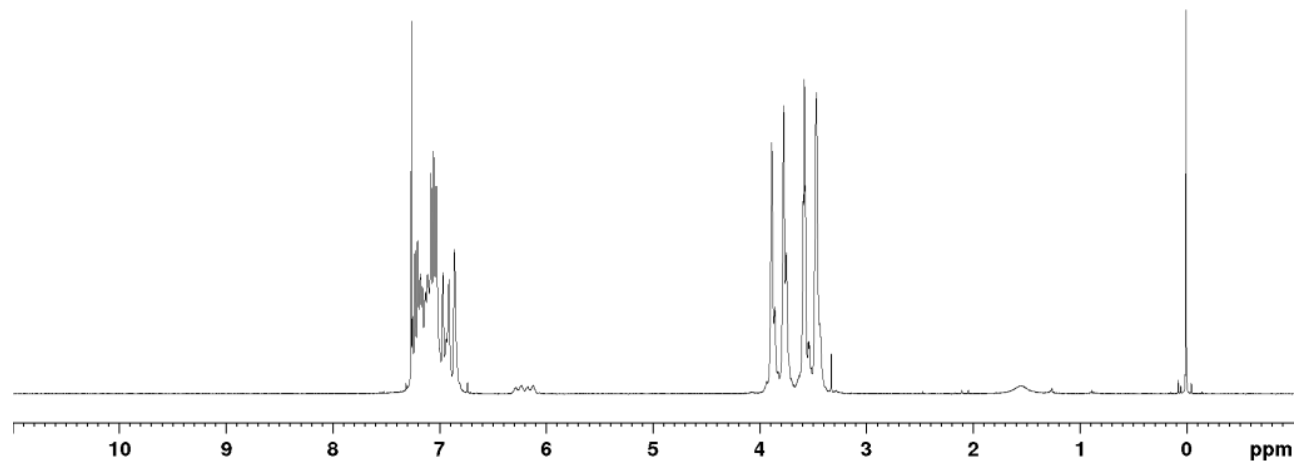

${ }^{19} \mathrm{~F} \mathrm{NMR}\left(\mathrm{CDCl}_{3}\right)$

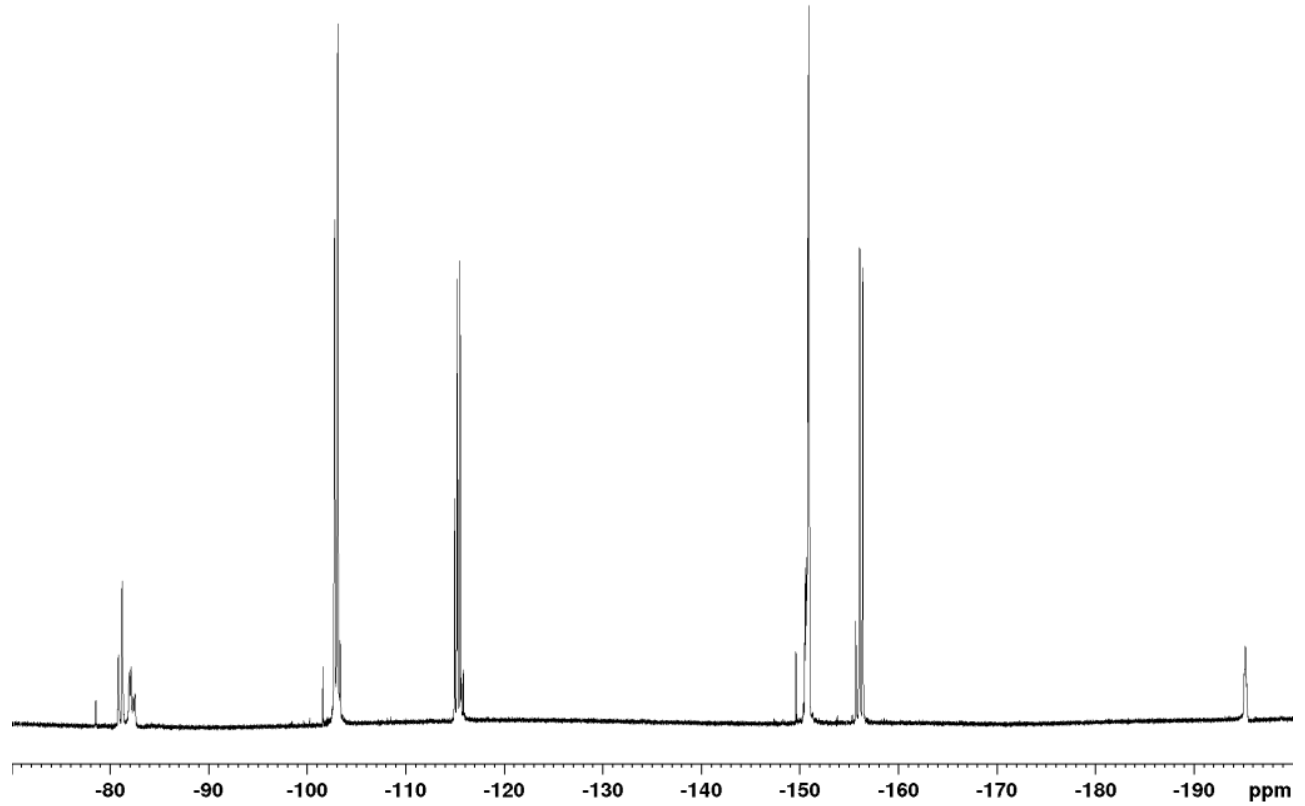


${ }^{13} \mathrm{C} \mathrm{NMR}\left(\mathrm{CDCl}_{3}\right)$

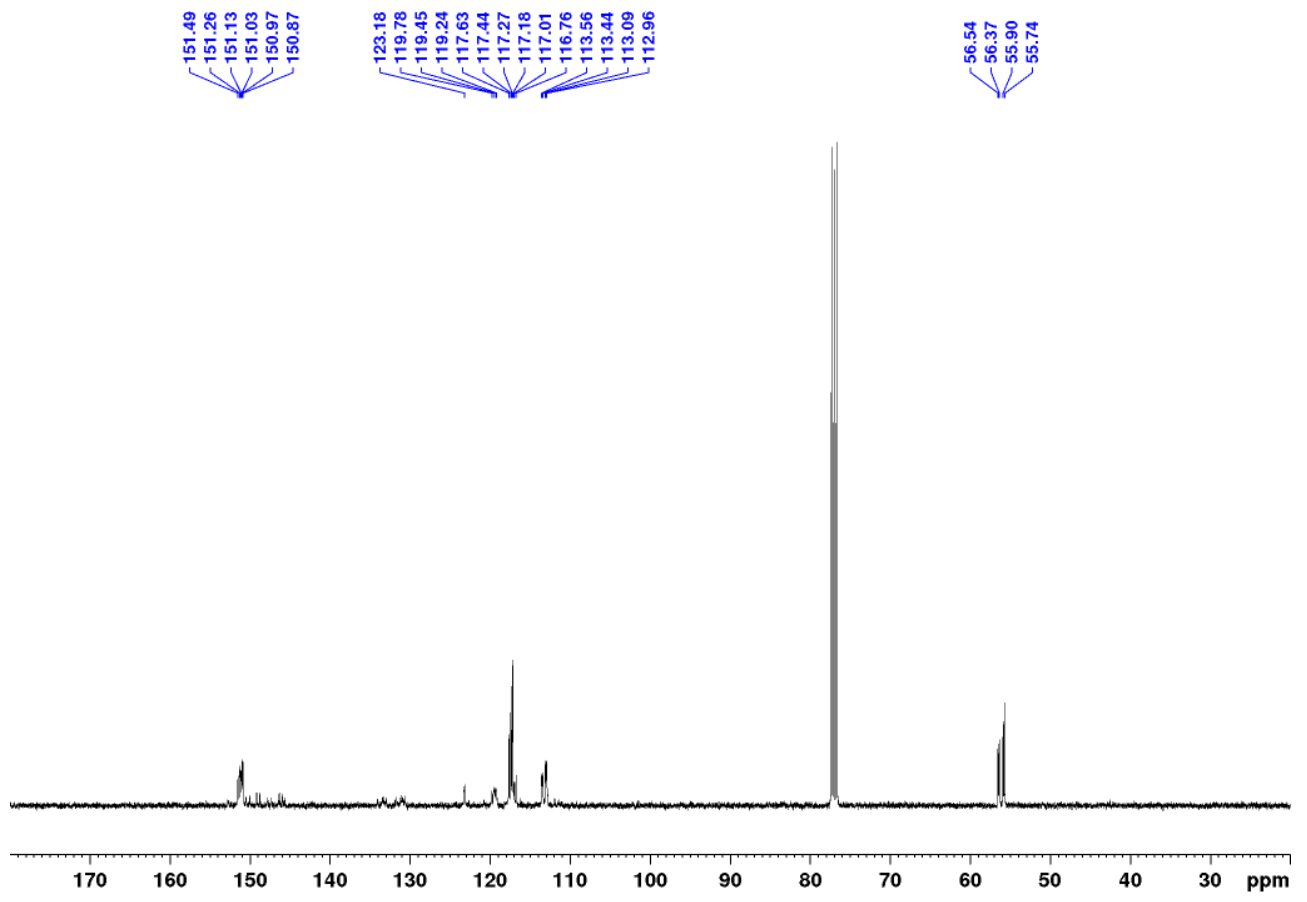


TGA

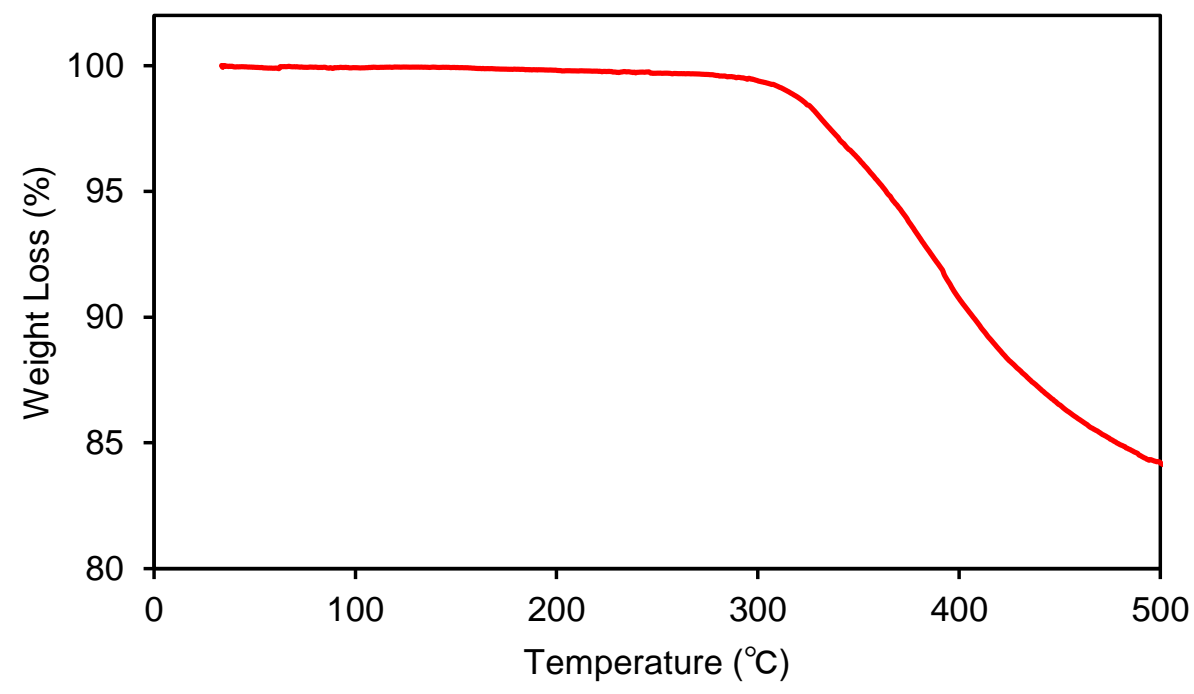

DSC

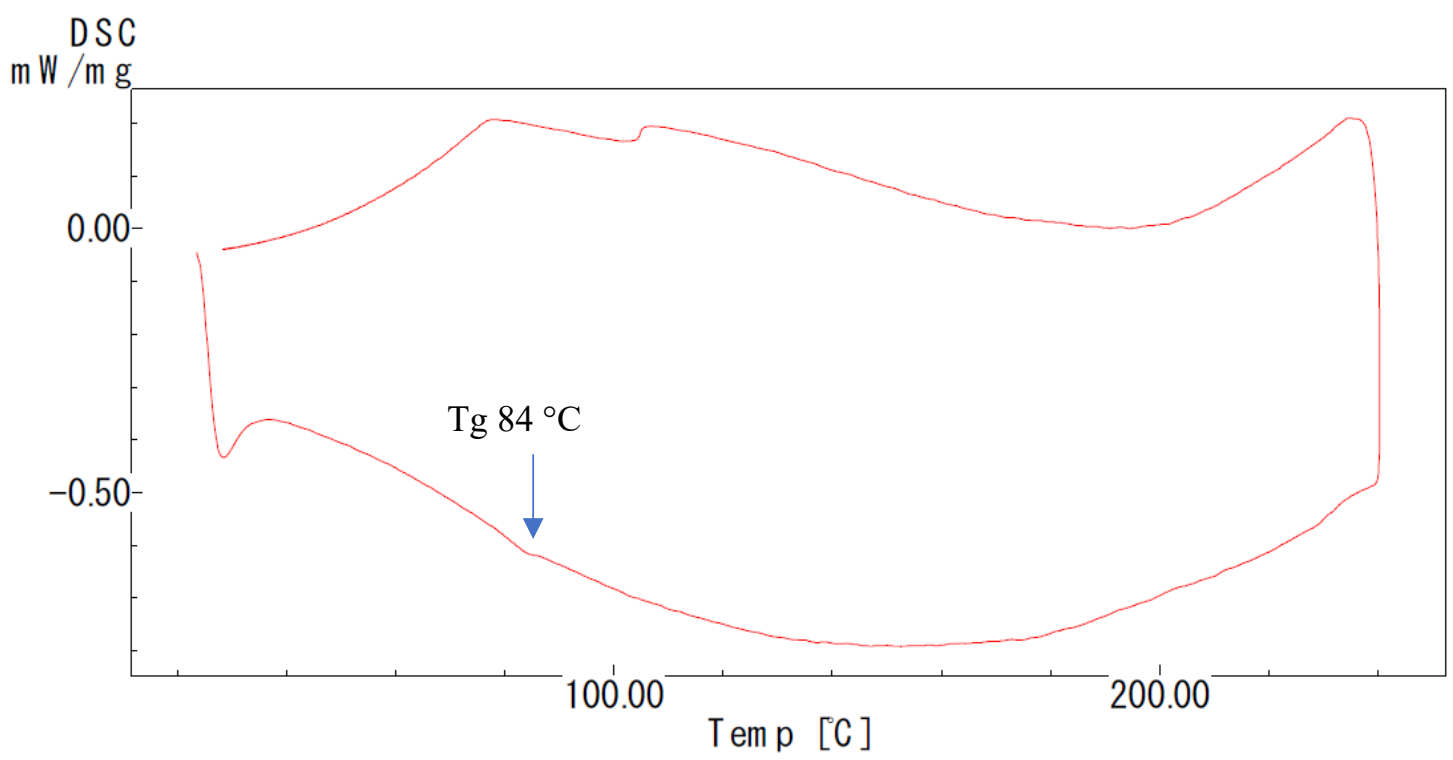


${ }^{1} \mathrm{H} \mathrm{NMR}\left(\mathrm{CDCl}_{3}\right)$<smiles>COc1cc(/C(F)=C(\F)C(C)(C)C)c(OC)cc1/C(F)=C(\F)Oc1ccc(C(C)(C)c2ccc(OC(C)(C)CC3CC4CCCC(C)(C4)C3)cc2)cc1</smiles>

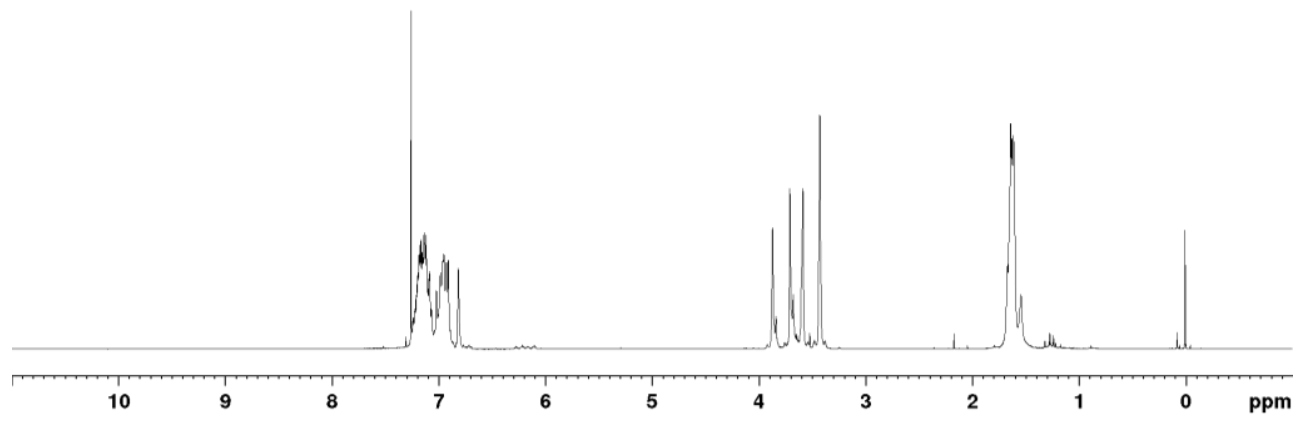

${ }^{19} \mathrm{~F} \mathrm{NMR}\left(\mathrm{CDCl}_{3}\right)$

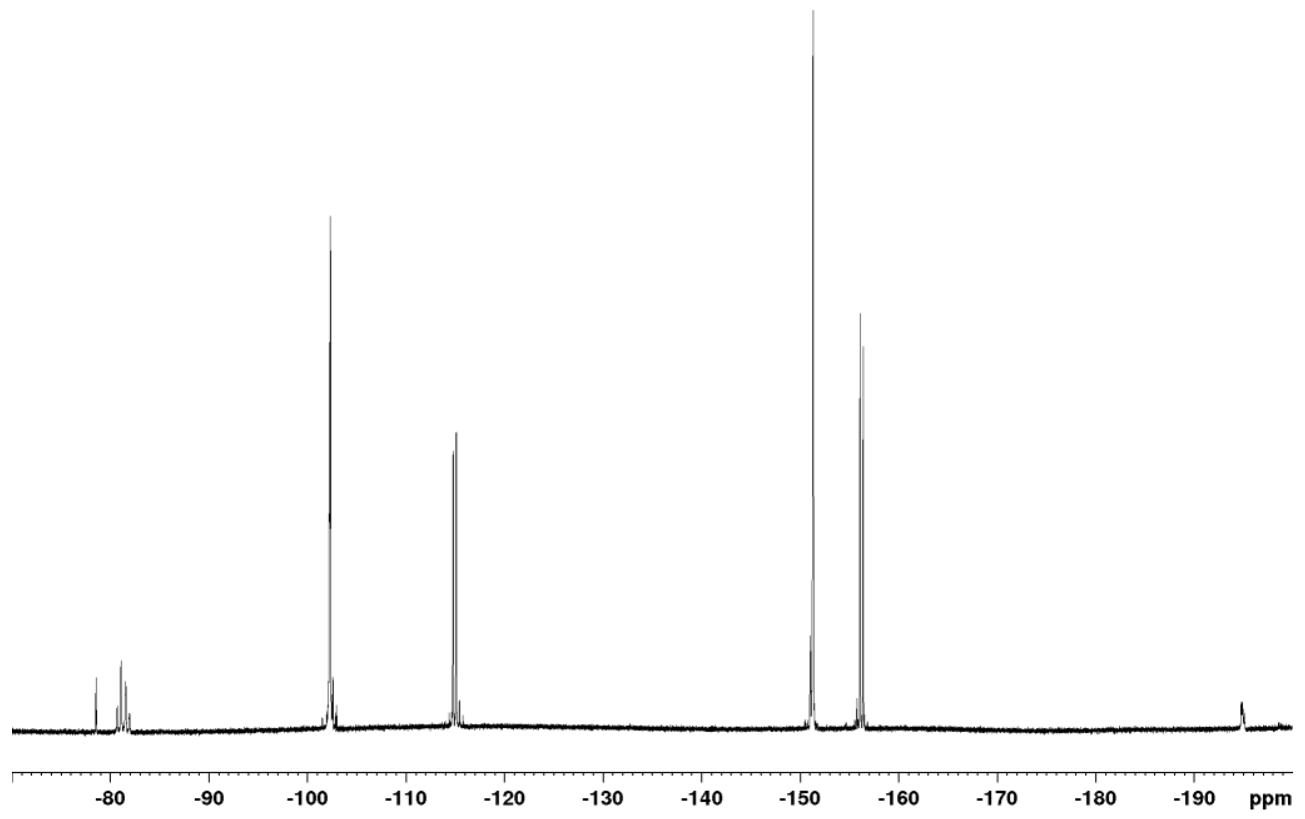


${ }^{13} \mathrm{C} \mathrm{NMR}\left(\mathrm{CDCl}_{3}\right)$

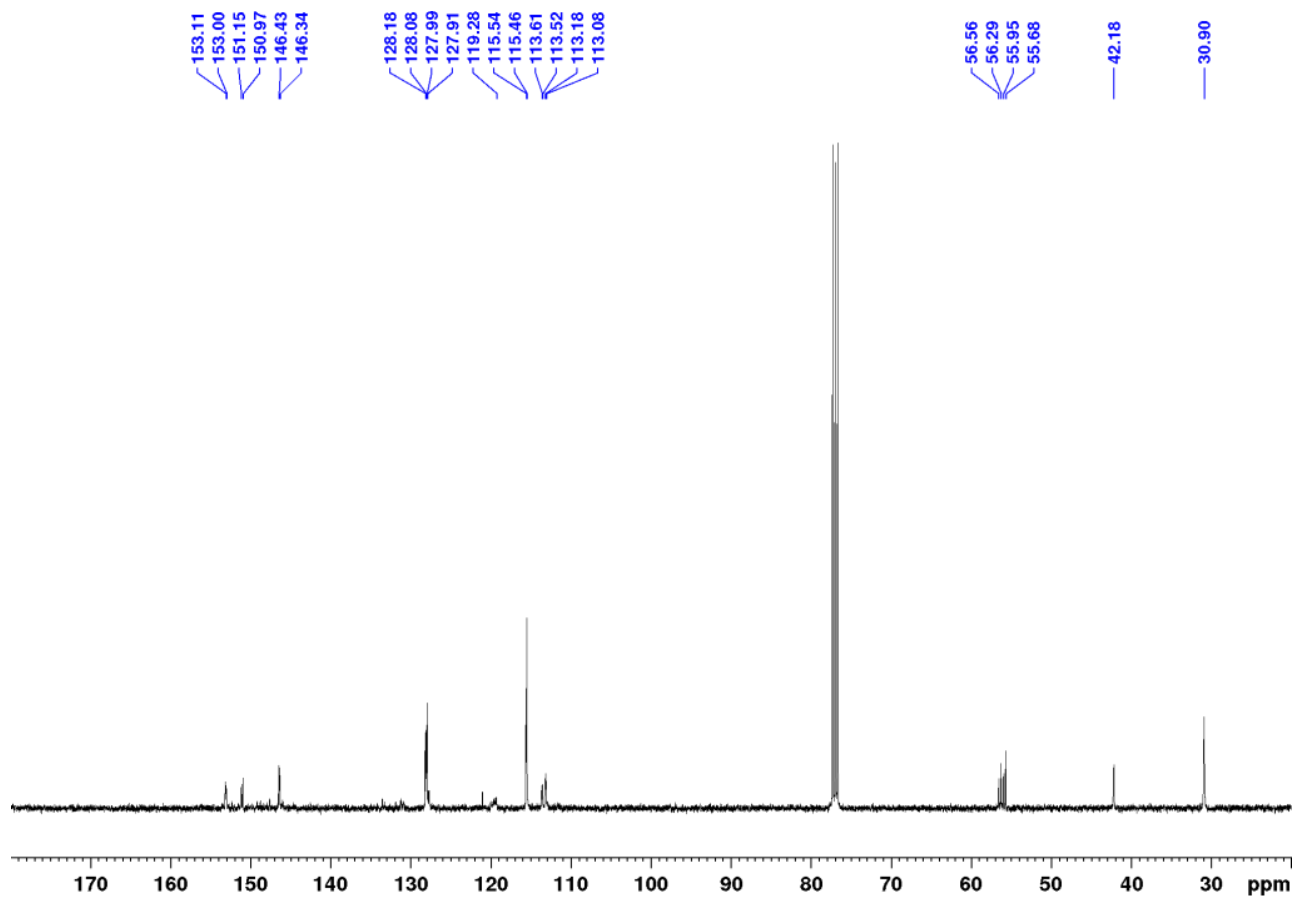


TGA

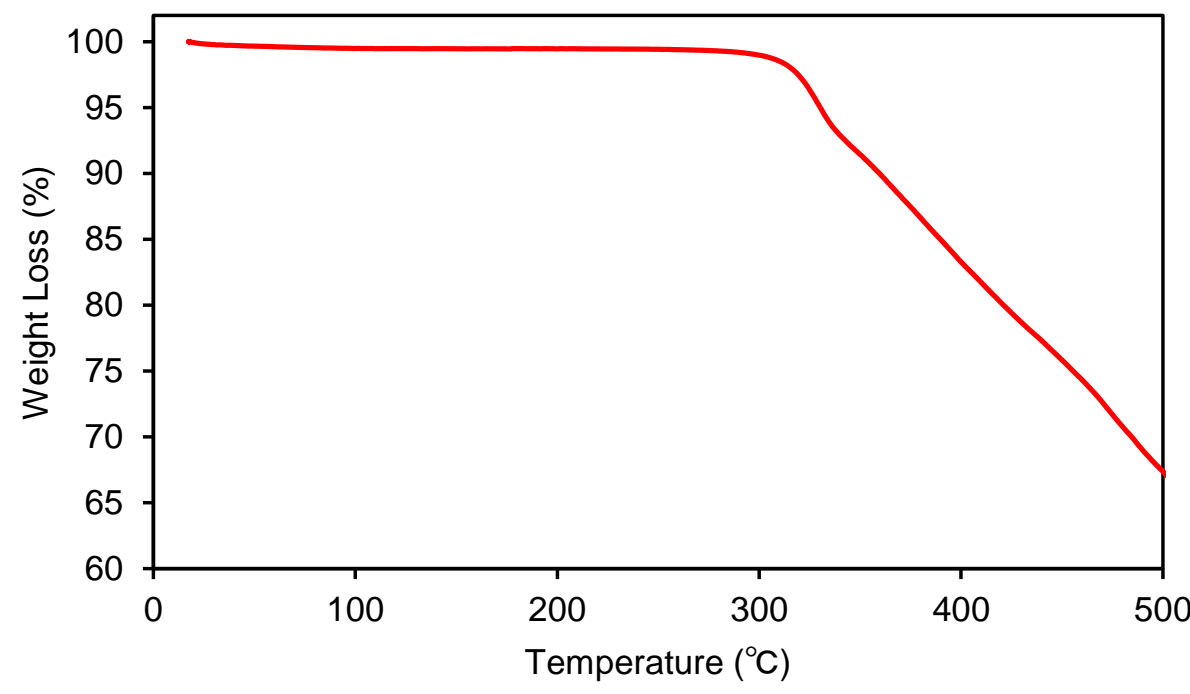

DSC

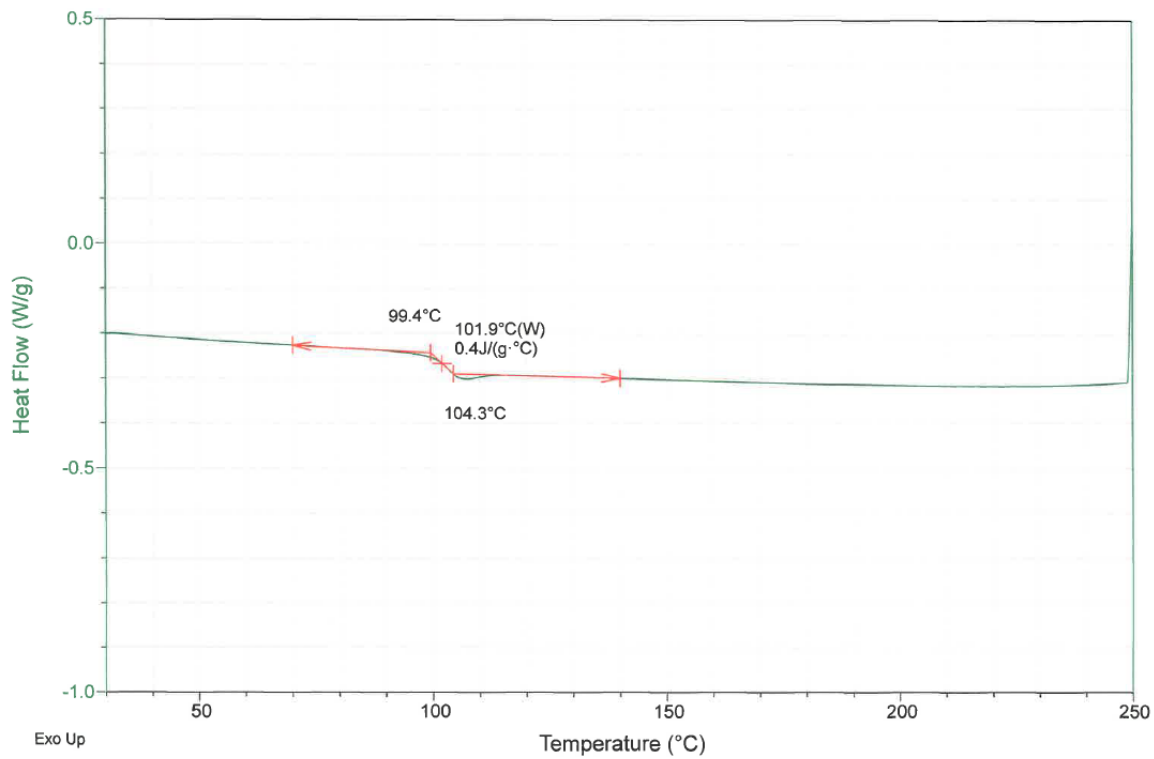


$3 c$

${ }^{1} \mathrm{H} \mathrm{NMR}\left(\mathrm{CDCl}_{3}\right)$

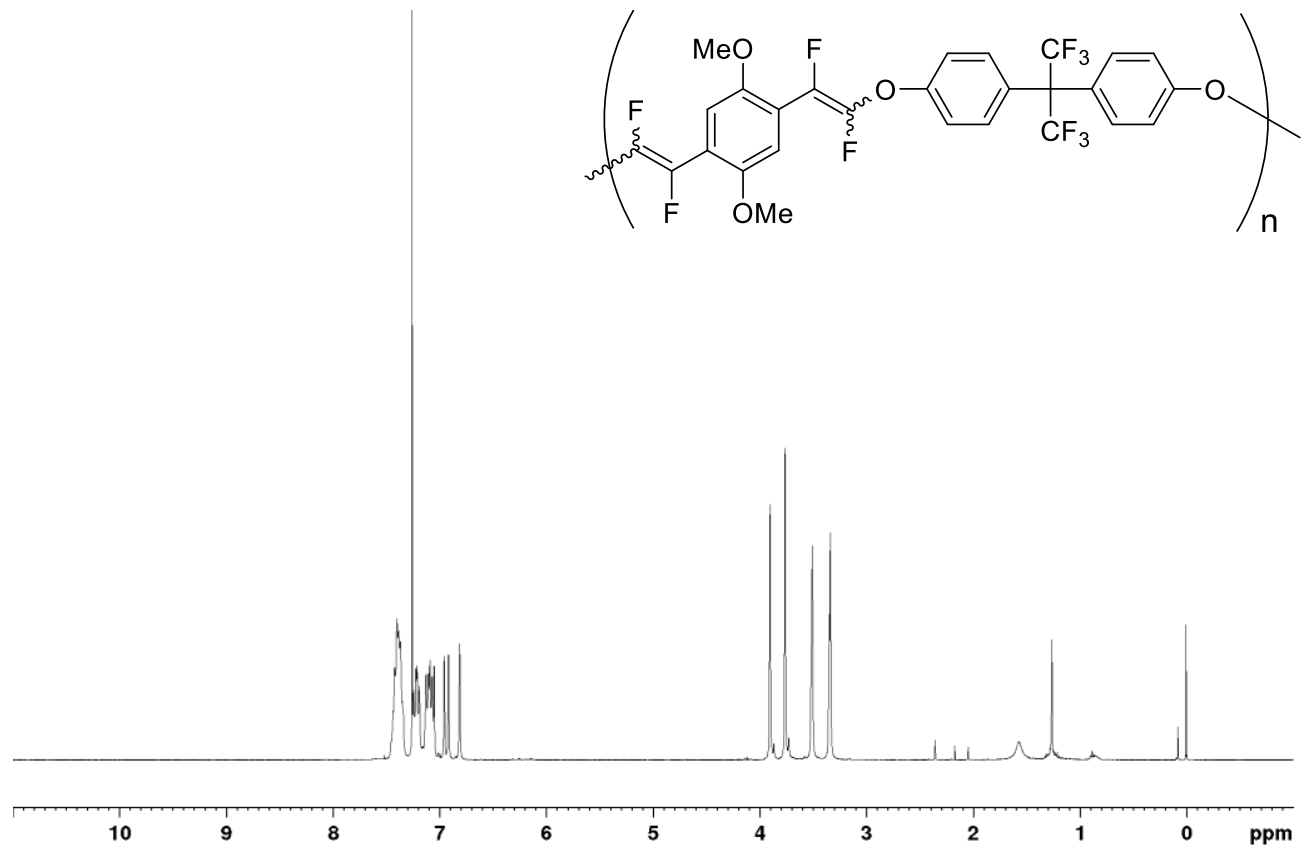

${ }^{19} \mathrm{~F} \mathrm{NMR}\left(\mathrm{CDCl}_{3}\right)$

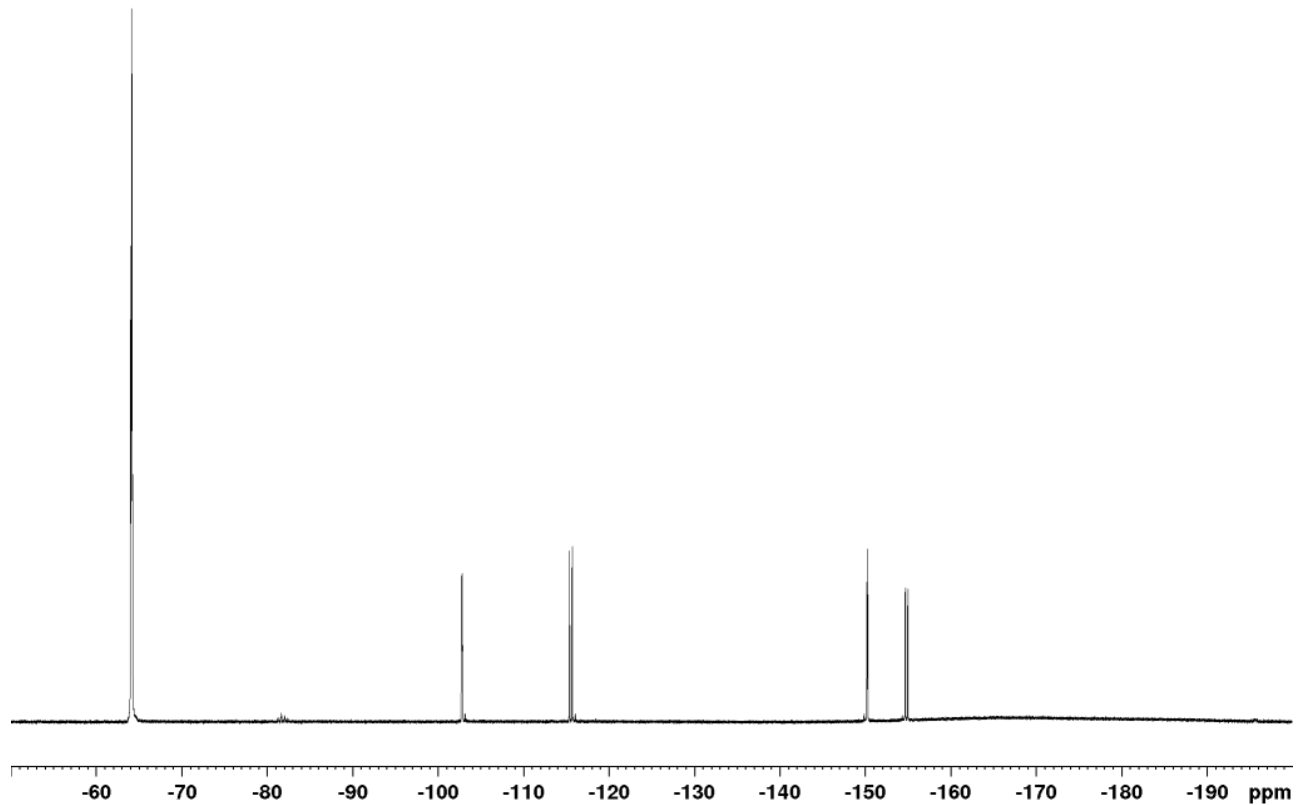


${ }^{13} \mathrm{C} \mathrm{NMR}\left(\mathrm{CDCl}_{3}\right)$

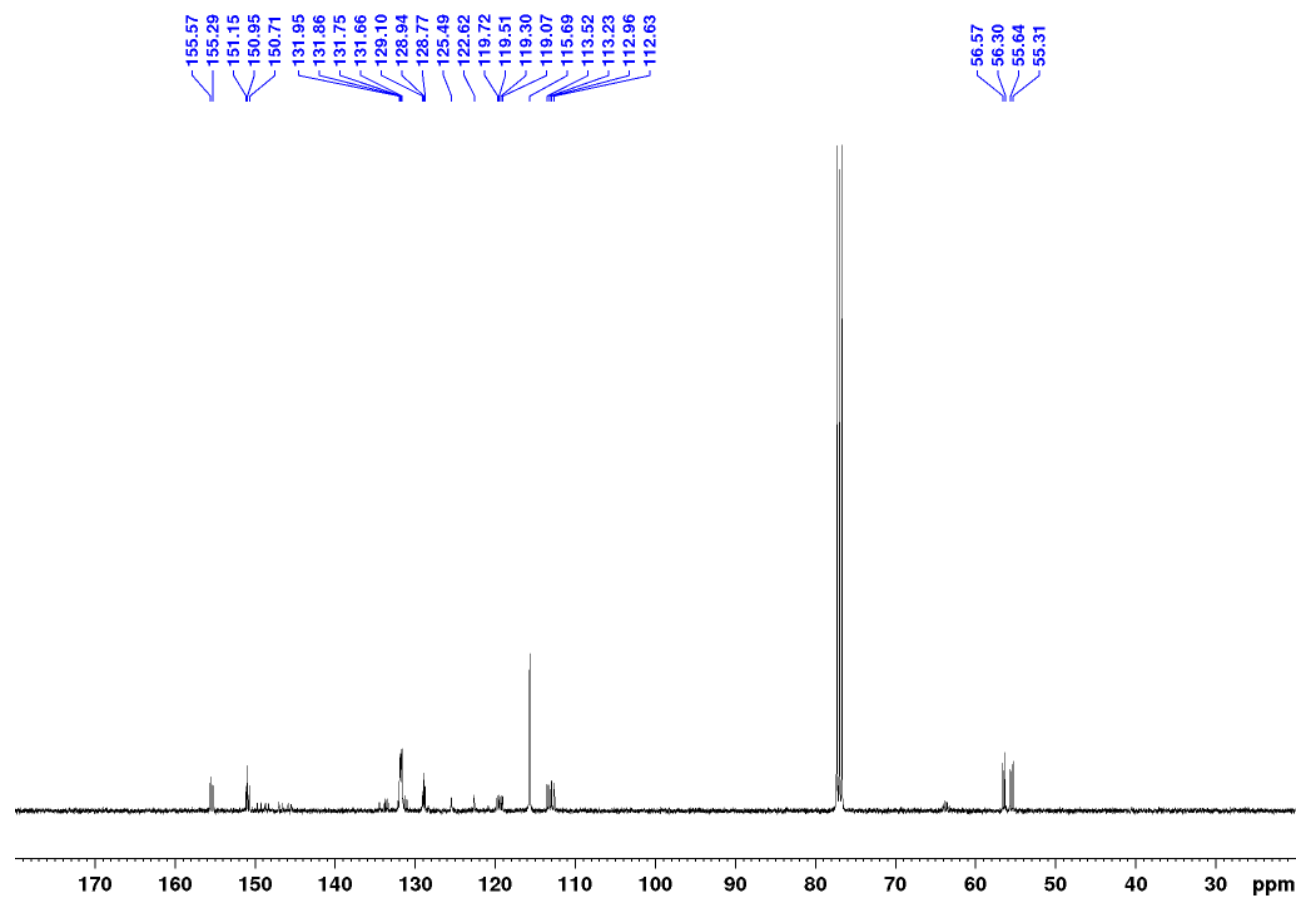


TGA

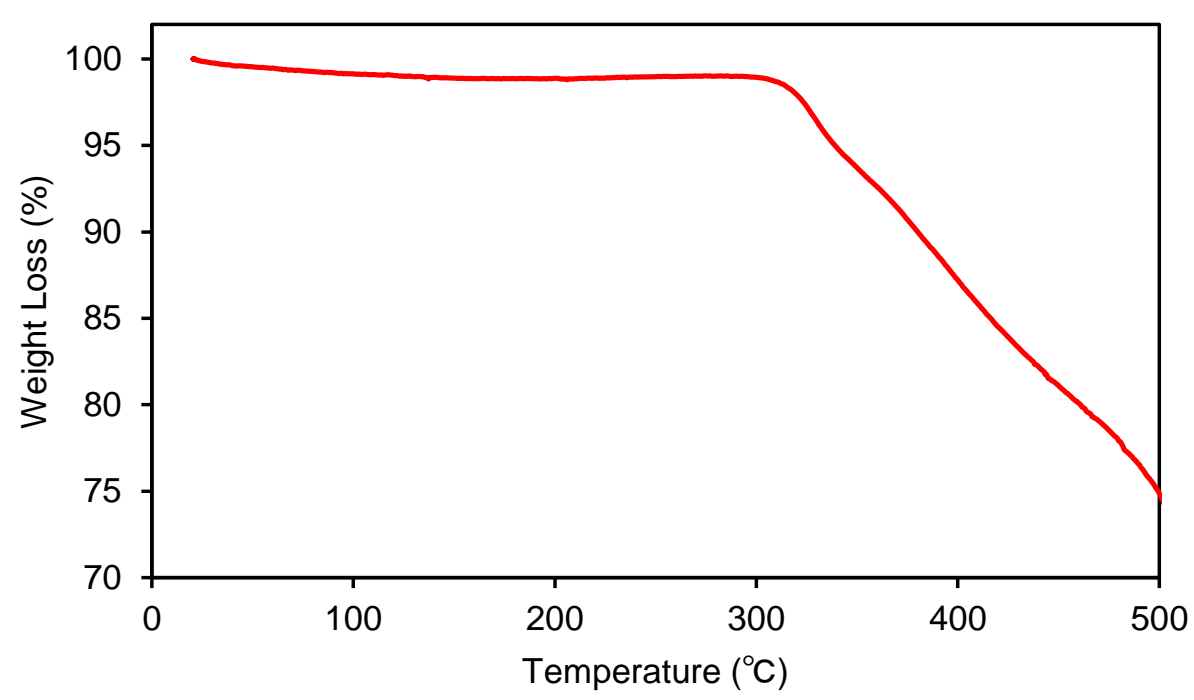

DSC

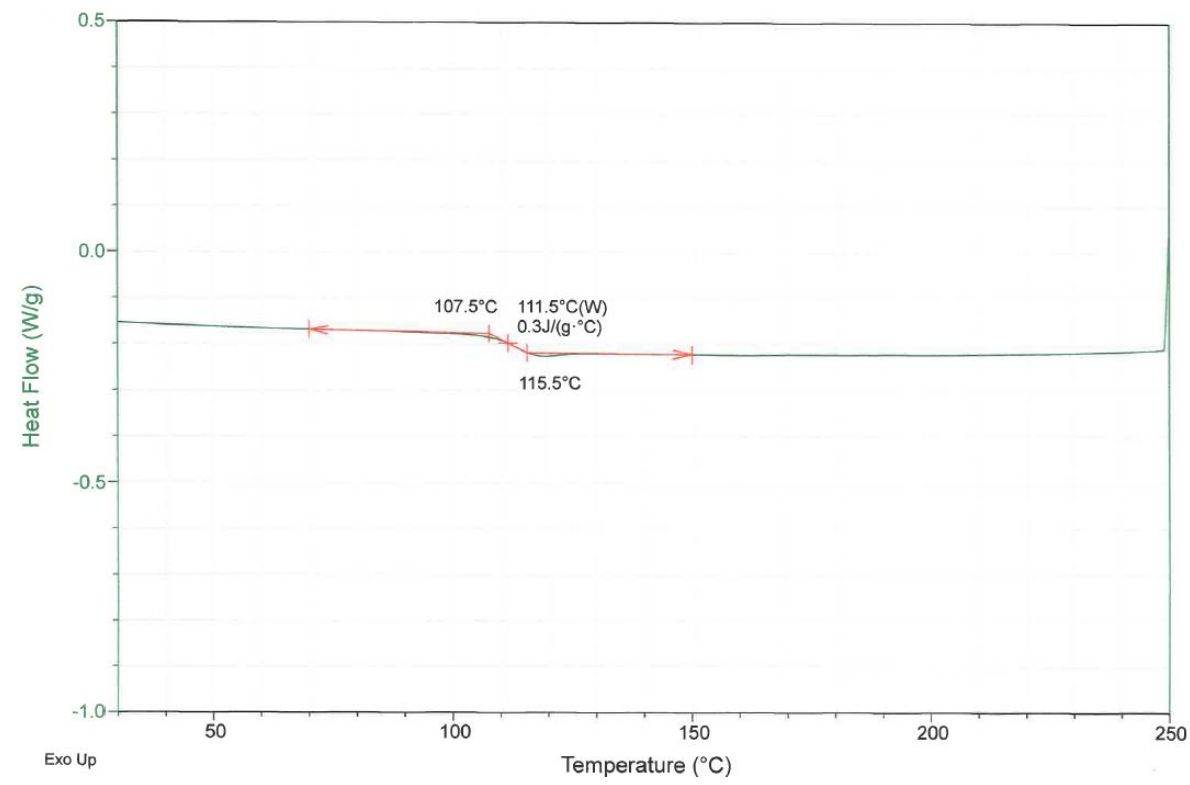


${ }^{1} \mathrm{H} \mathrm{NMR}\left(\mathrm{CDCl}_{3}\right)$<smiles>COc1cc(/C(F)=C(\F)C(C)(C)C)c(OC)cc1/C(F)=C(\F)Oc1ccc(Oc2ccc(OC(C)(C)C)cc2)cc1</smiles>

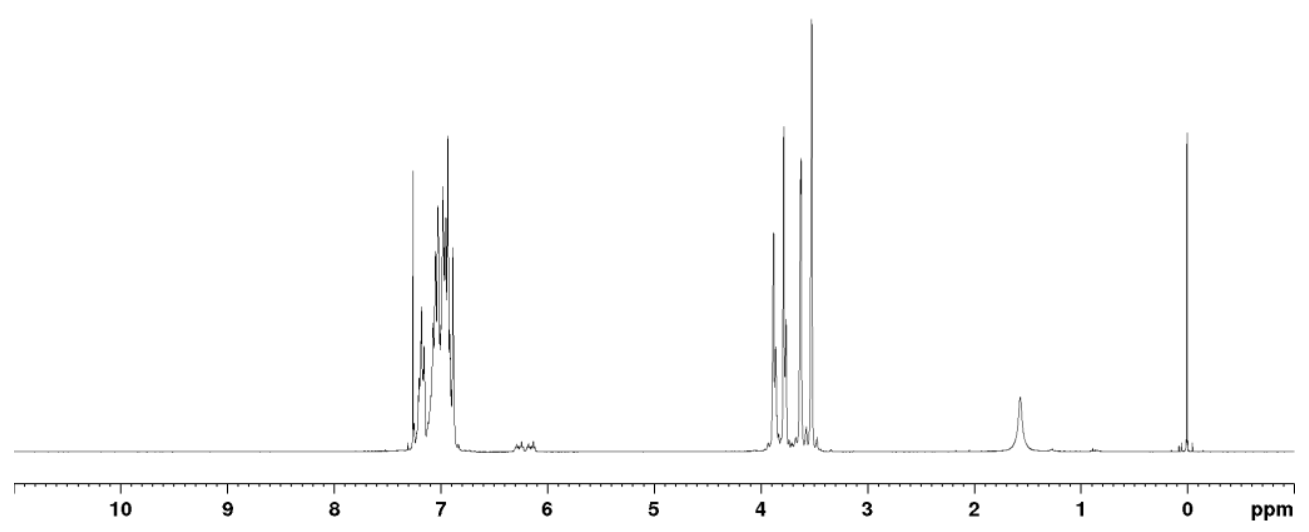

${ }^{19} \mathrm{~F} \mathrm{NMR}\left(\mathrm{CDCl}_{3}\right)$

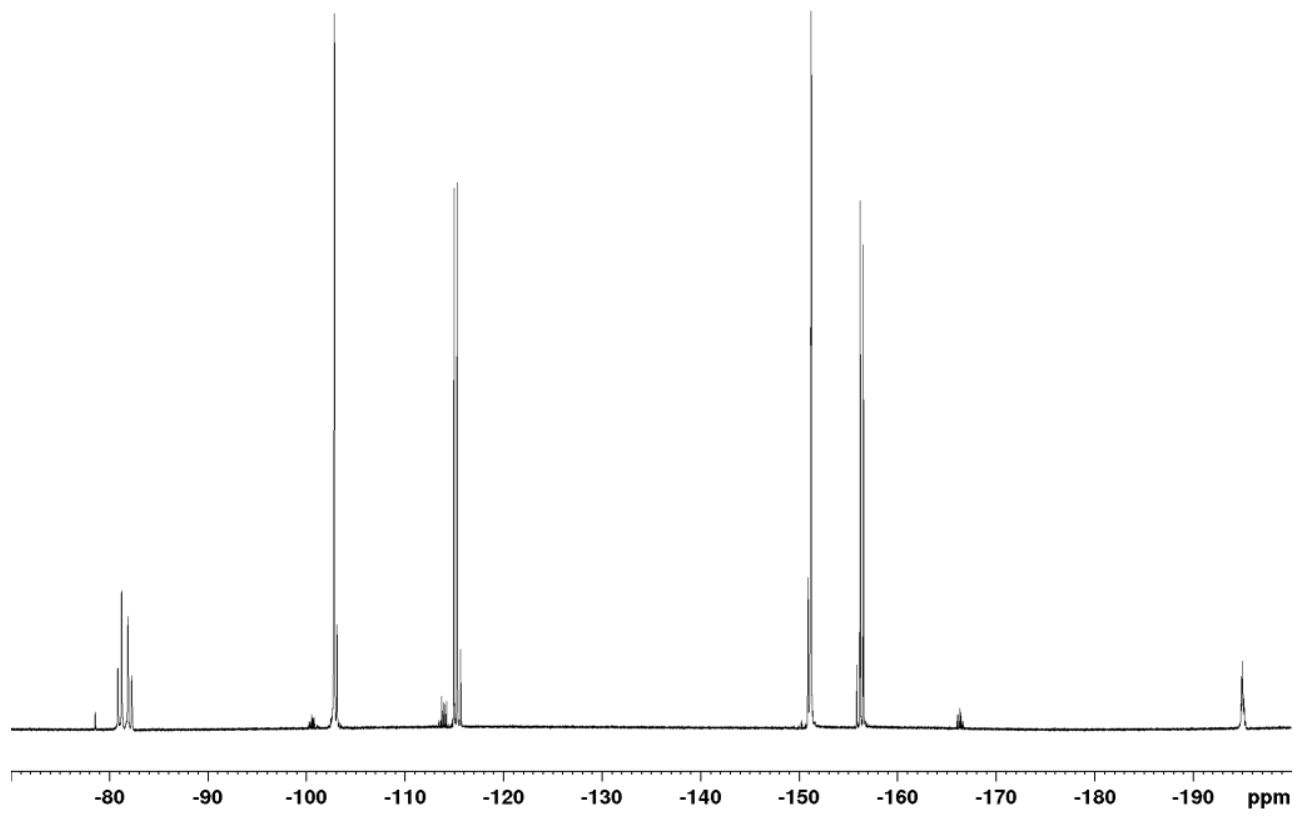


${ }^{13} \mathrm{C} \mathrm{NMR}\left(\mathrm{CDCl}_{3}\right)$

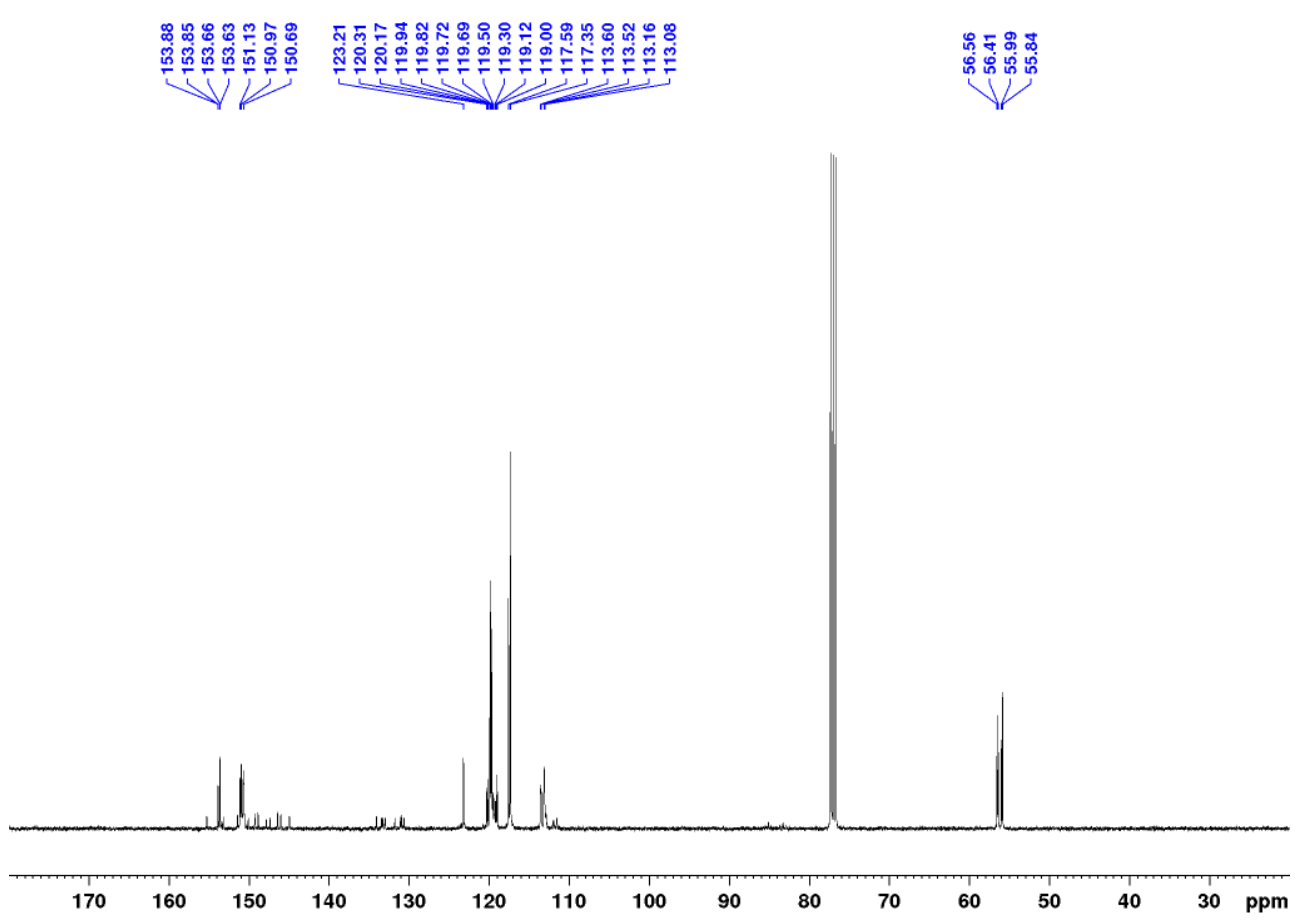


TGA

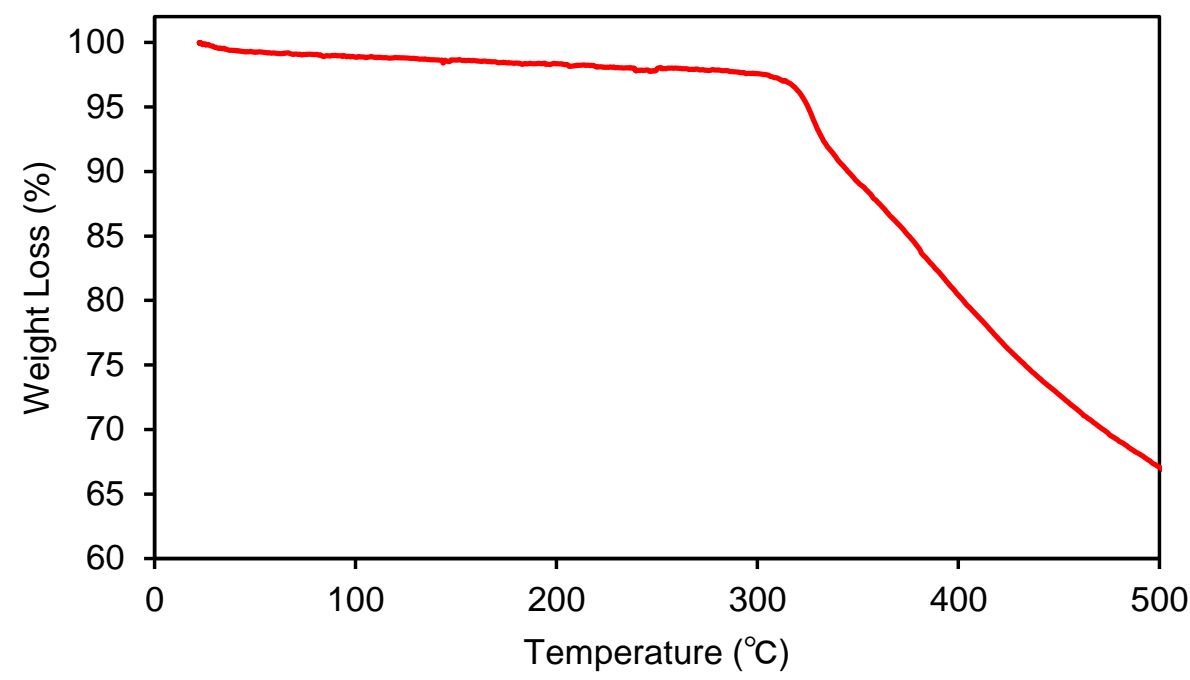

DSC

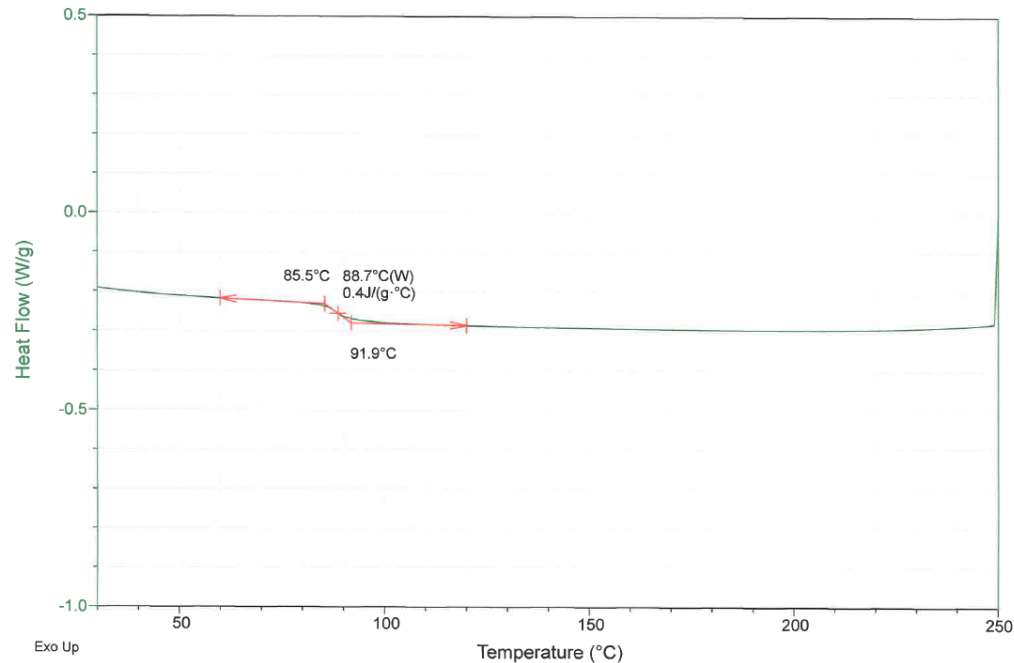


${ }^{1} \mathrm{H} \mathrm{NMR}\left(\mathrm{CDCl}_{3}\right)$

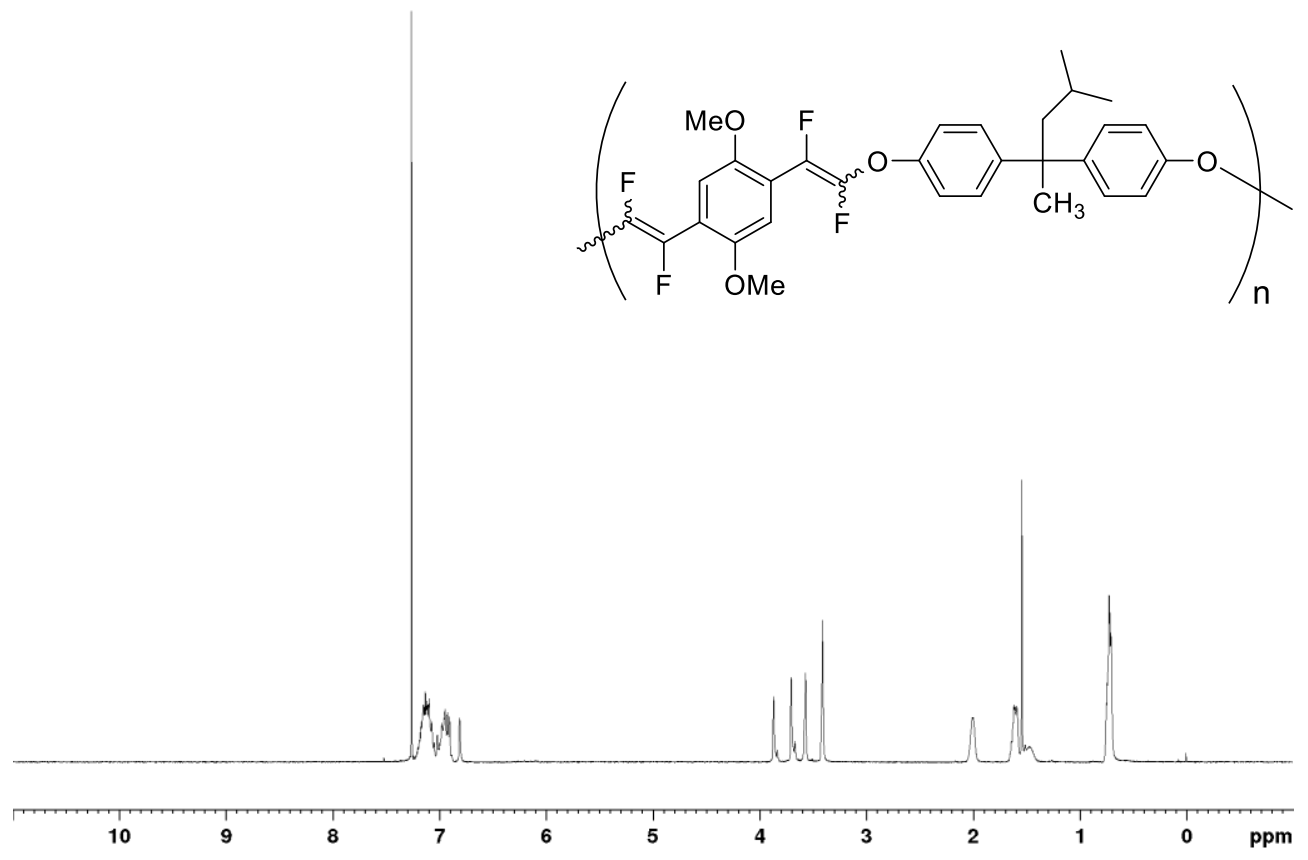

${ }^{19} \mathrm{~F} \mathrm{NMR}\left(\mathrm{CDCl}_{3}\right)$

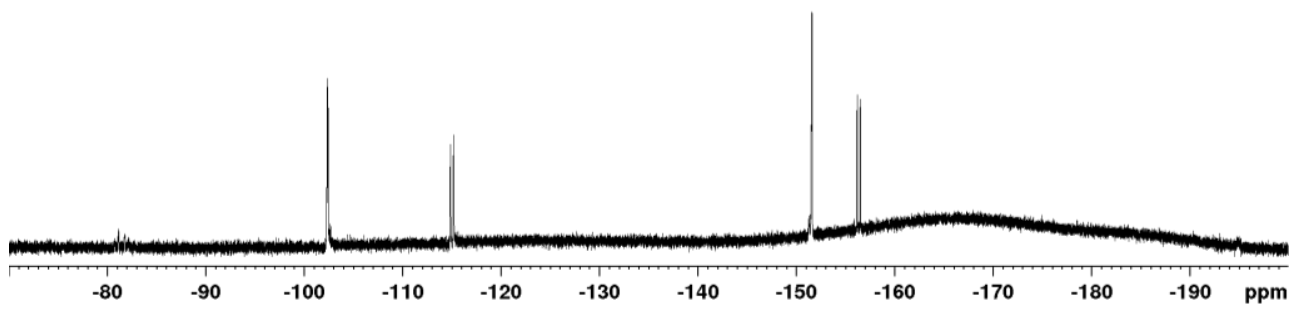


${ }^{13} \mathrm{C} \mathrm{NMR}\left(\mathrm{CDCl}_{3}\right)$

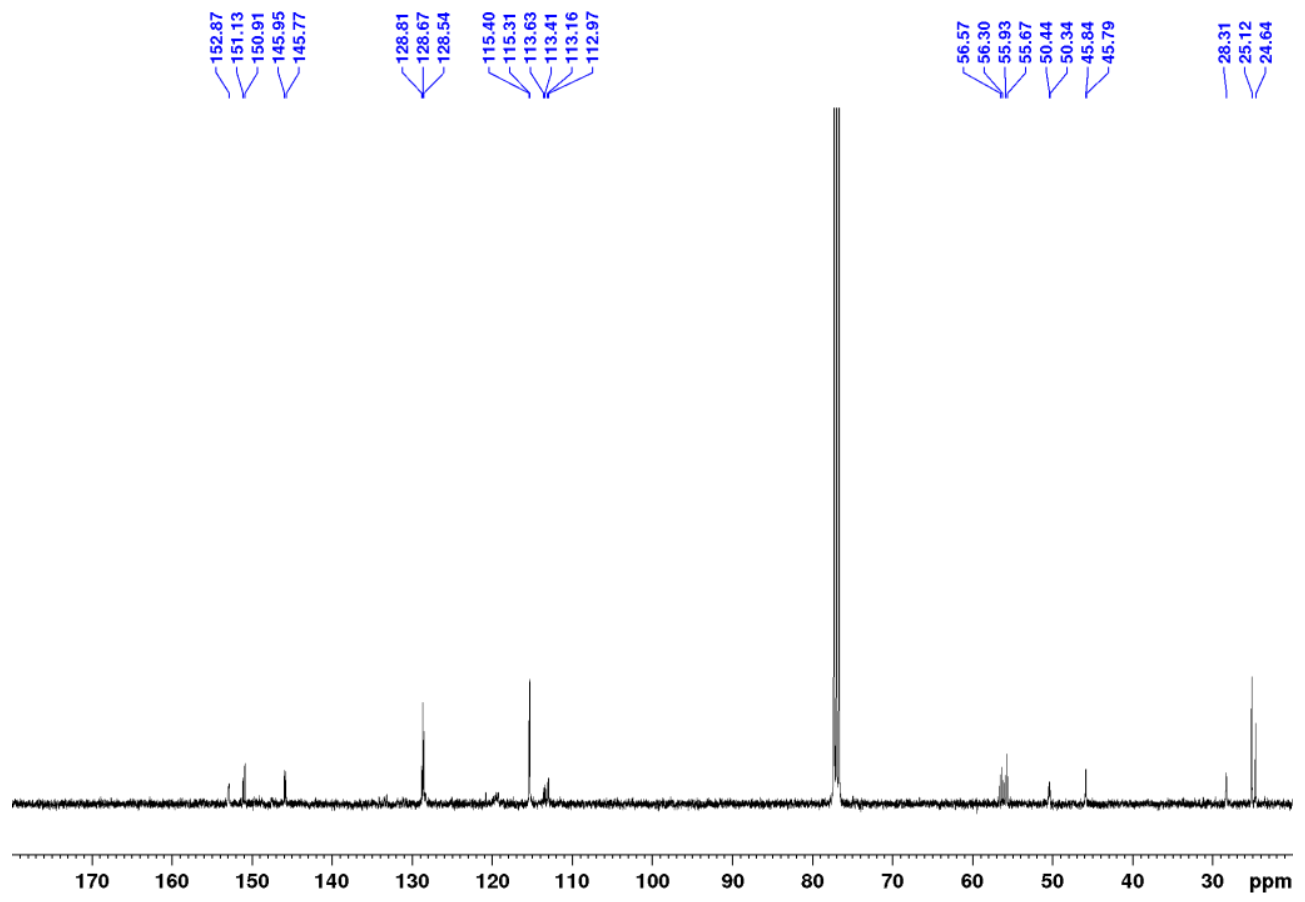


TGA

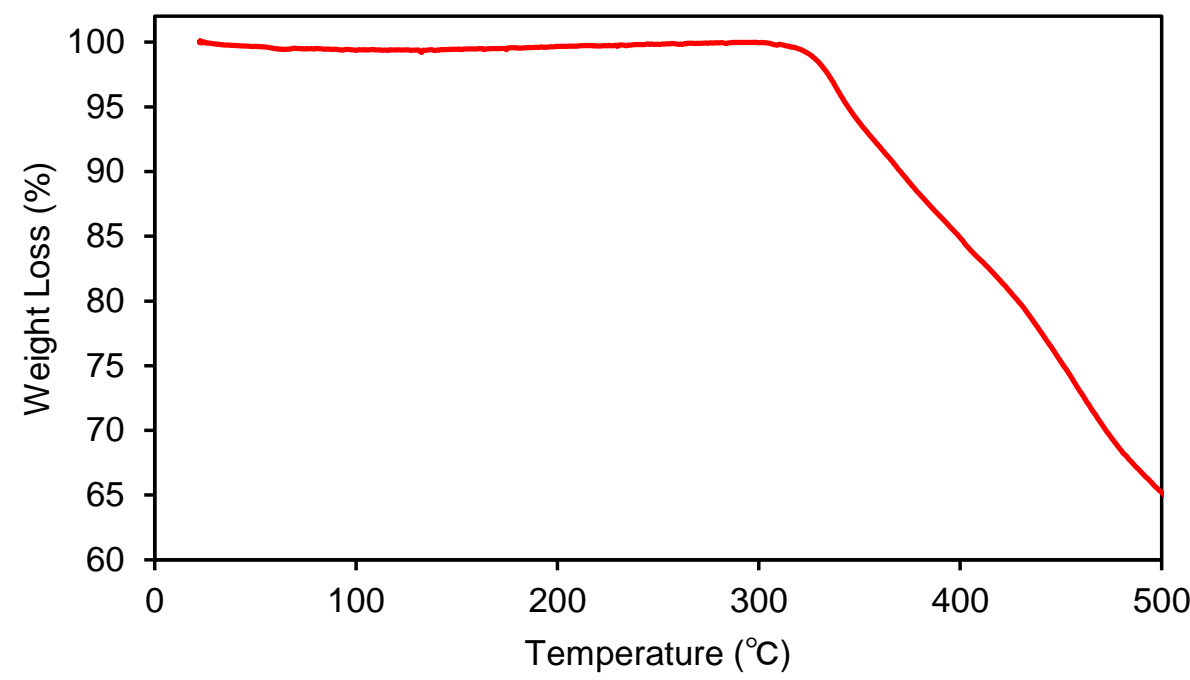

DSC

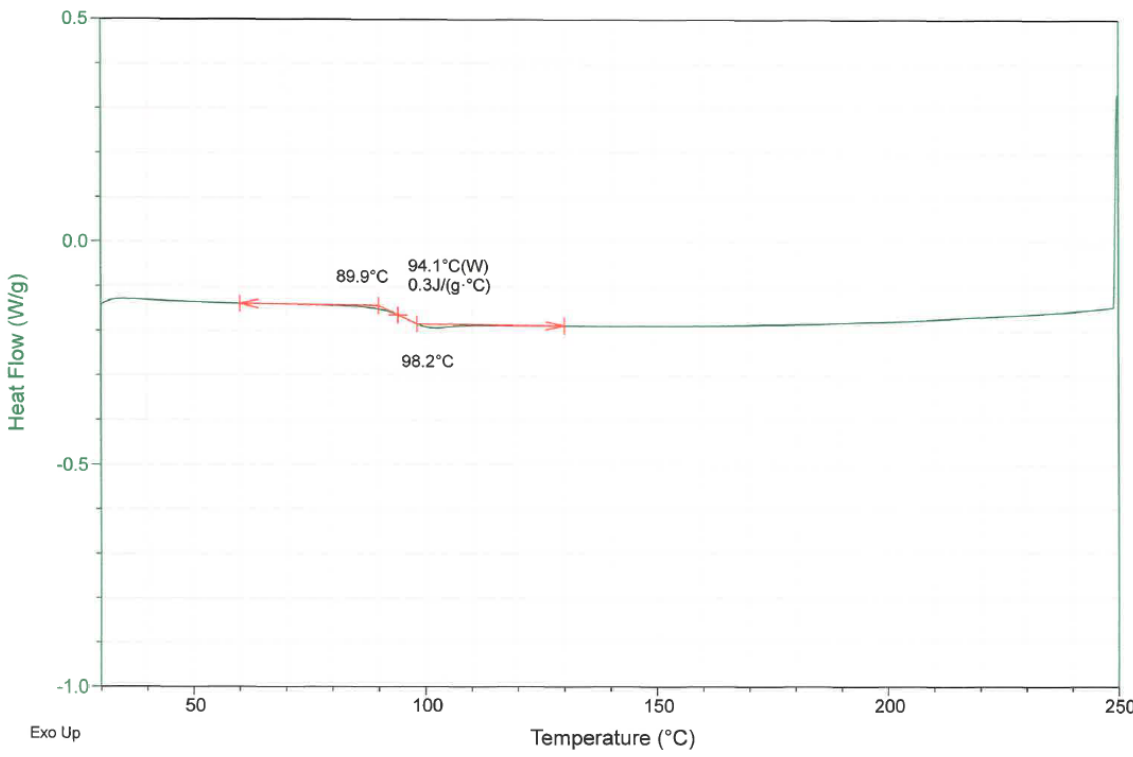


$3 g$

${ }^{1} \mathrm{H} \mathrm{NMR}\left(\mathrm{CDCl}_{3}\right)$

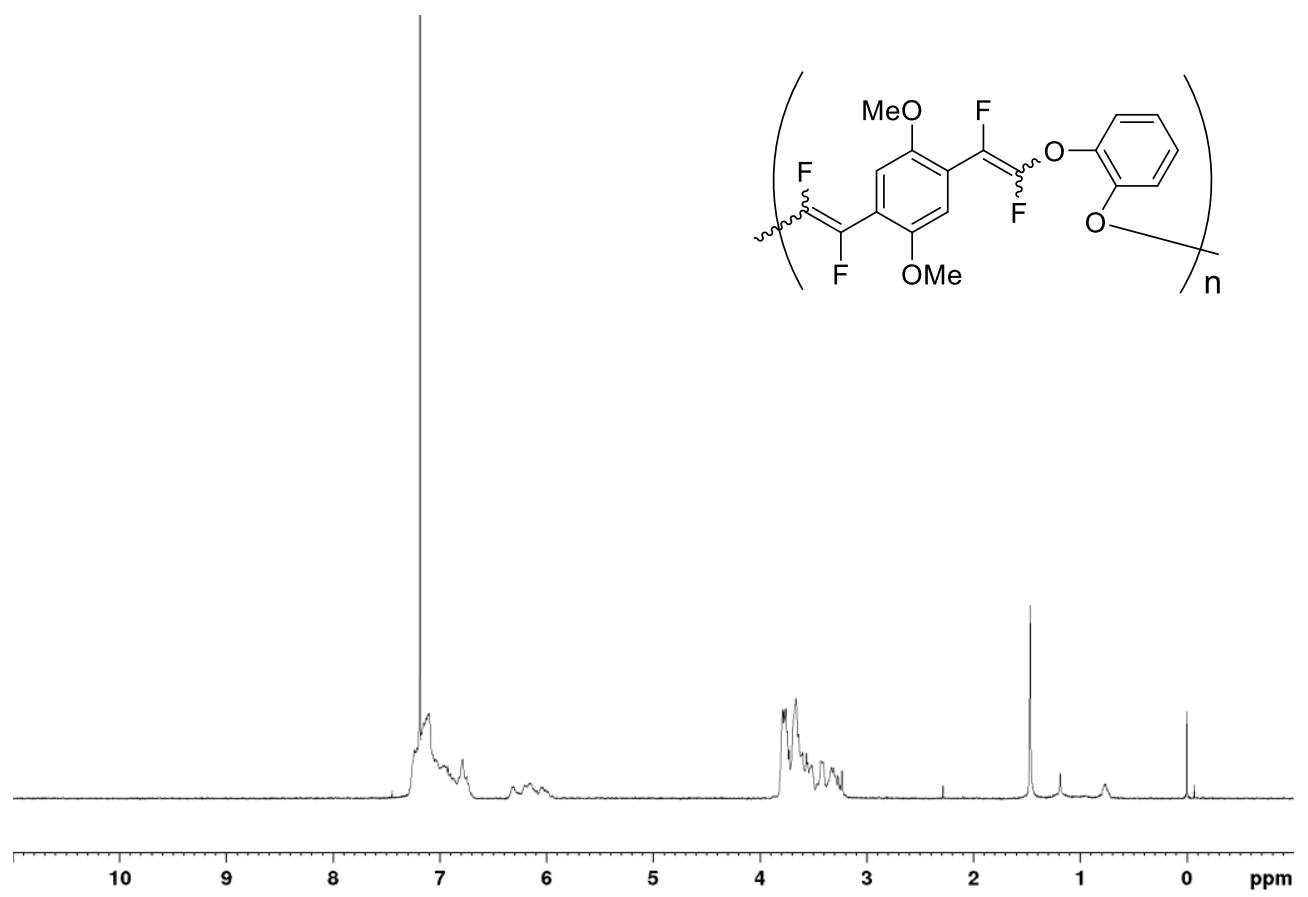

${ }^{19} \mathrm{~F} \mathrm{NMR}\left(\mathrm{CDCl}_{3}\right)$

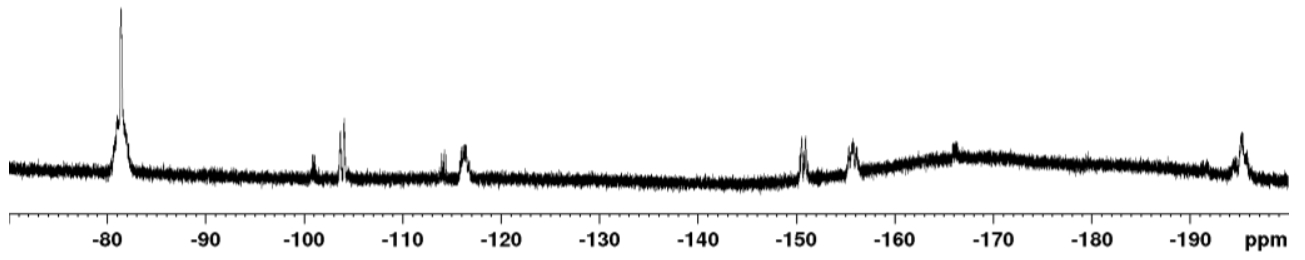


${ }^{13} \mathrm{C} \mathrm{NMR}\left(\mathrm{CDCl}_{3}\right)$

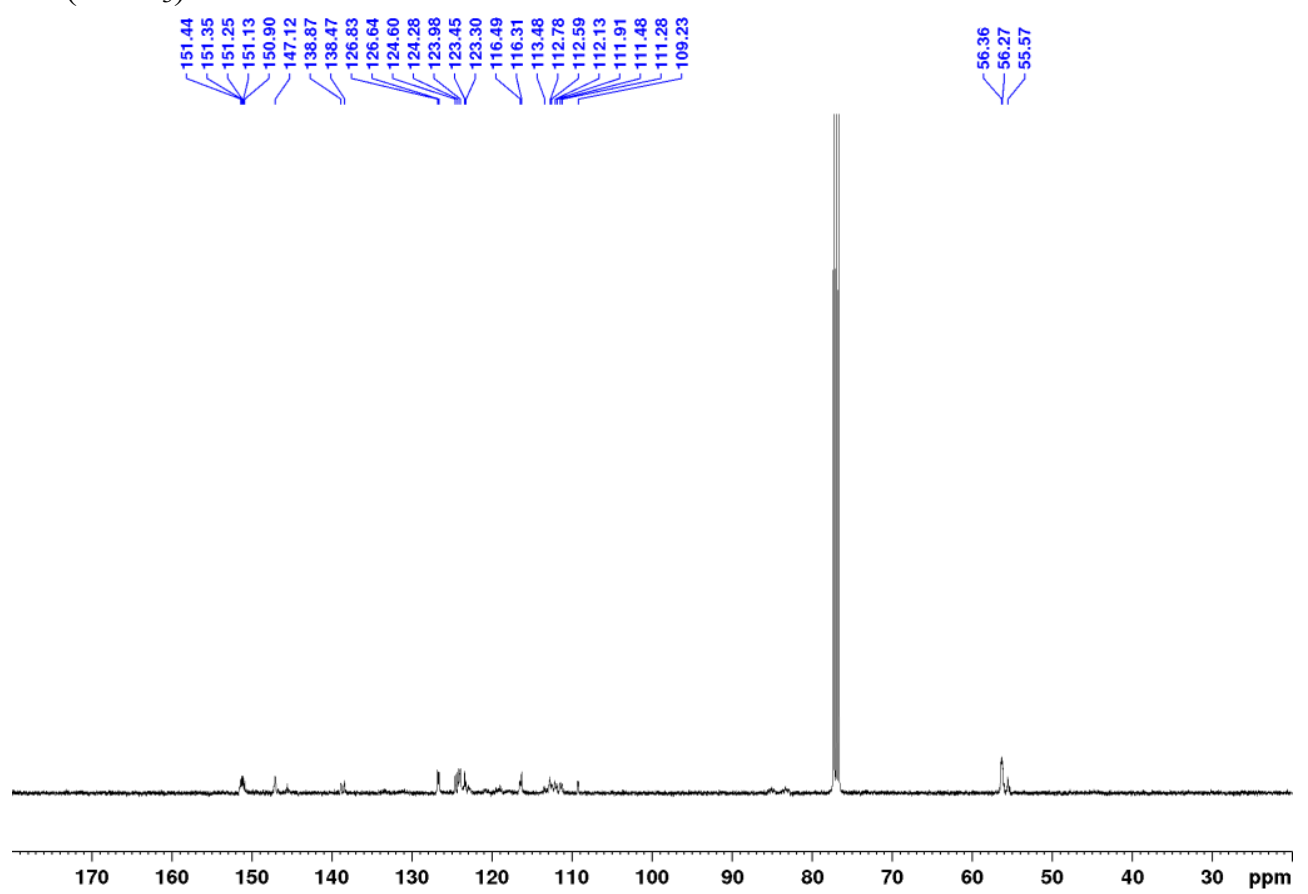


TGA

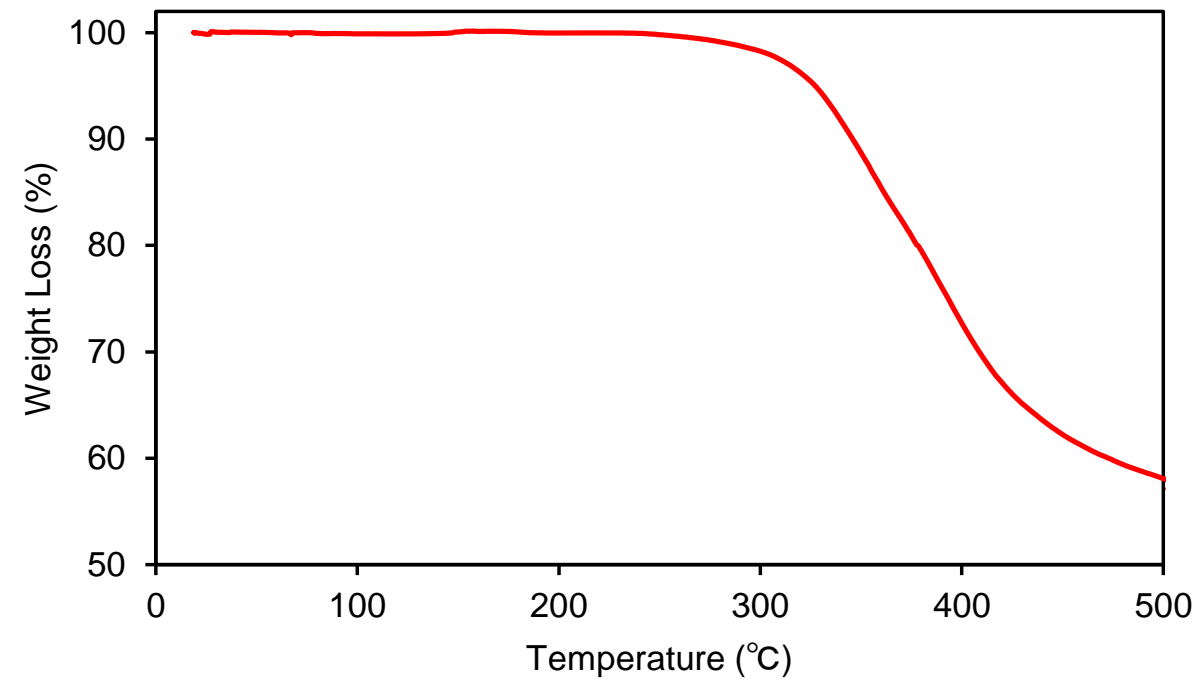

DSC

DSC

$\mathrm{mW} / \mathrm{m} \mathrm{g}$

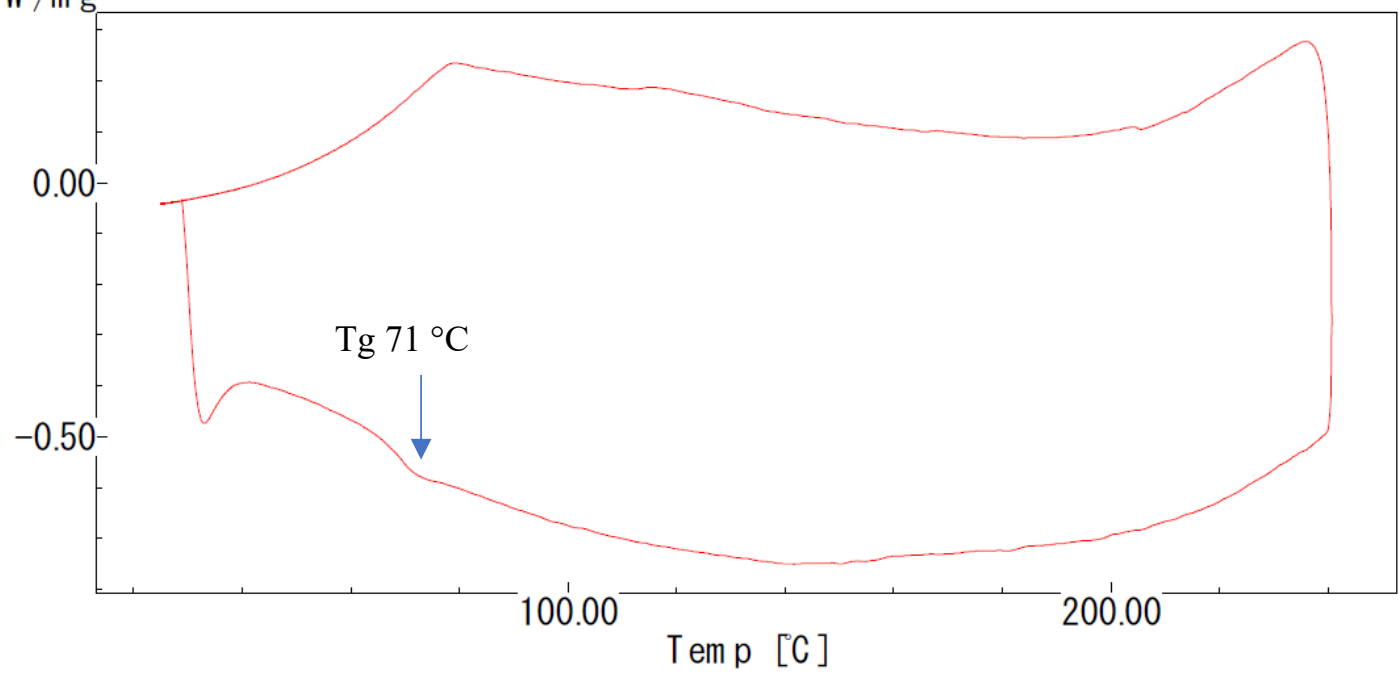


3h

${ }^{1} \mathrm{H} \mathrm{NMR}\left(\mathrm{CDCl}_{3}\right)$

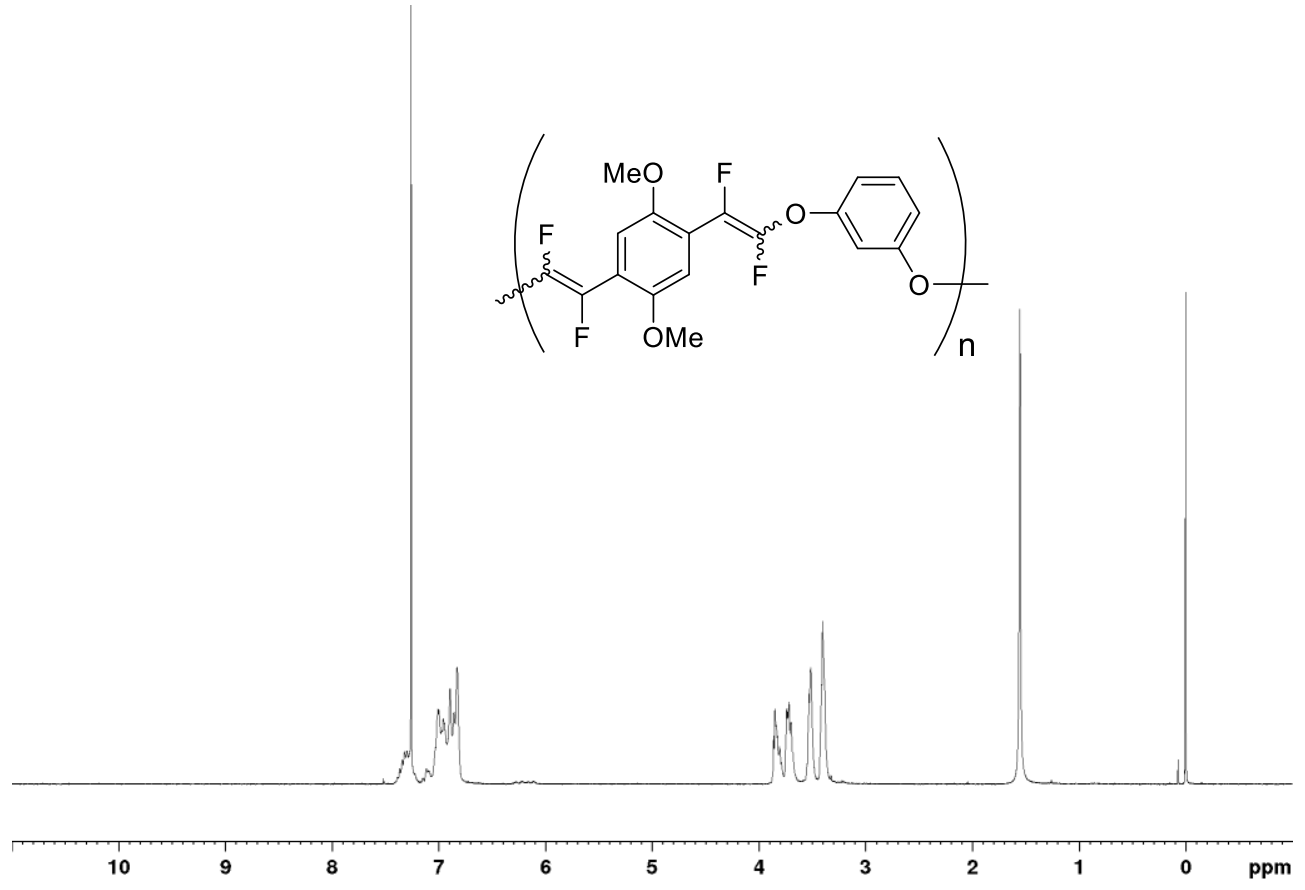

${ }^{19} \mathrm{~F} \mathrm{NMR}\left(\mathrm{CDCl}_{3}\right)$

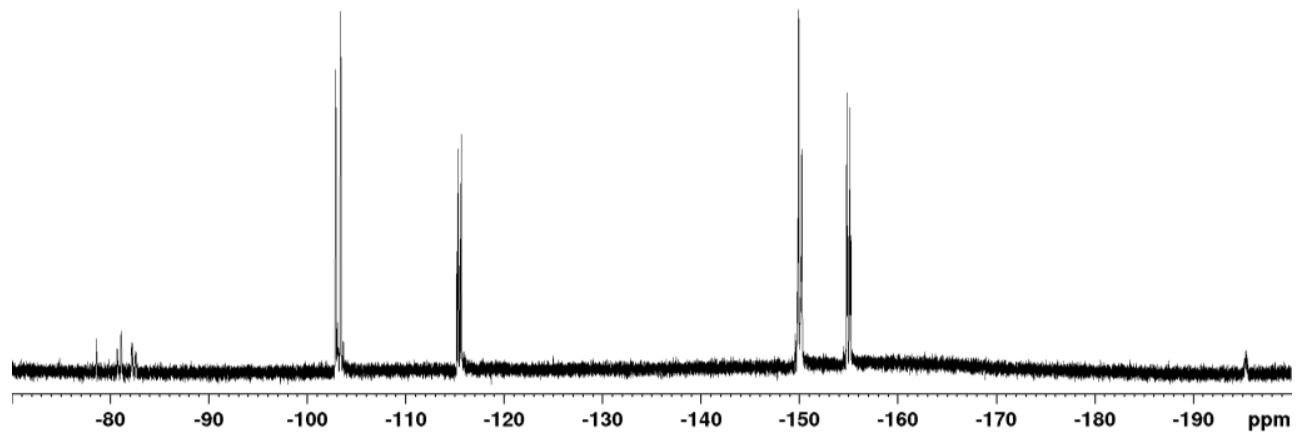


${ }^{13} \mathrm{C} \mathrm{NMR}\left(\mathrm{CDCl}_{3}\right)$

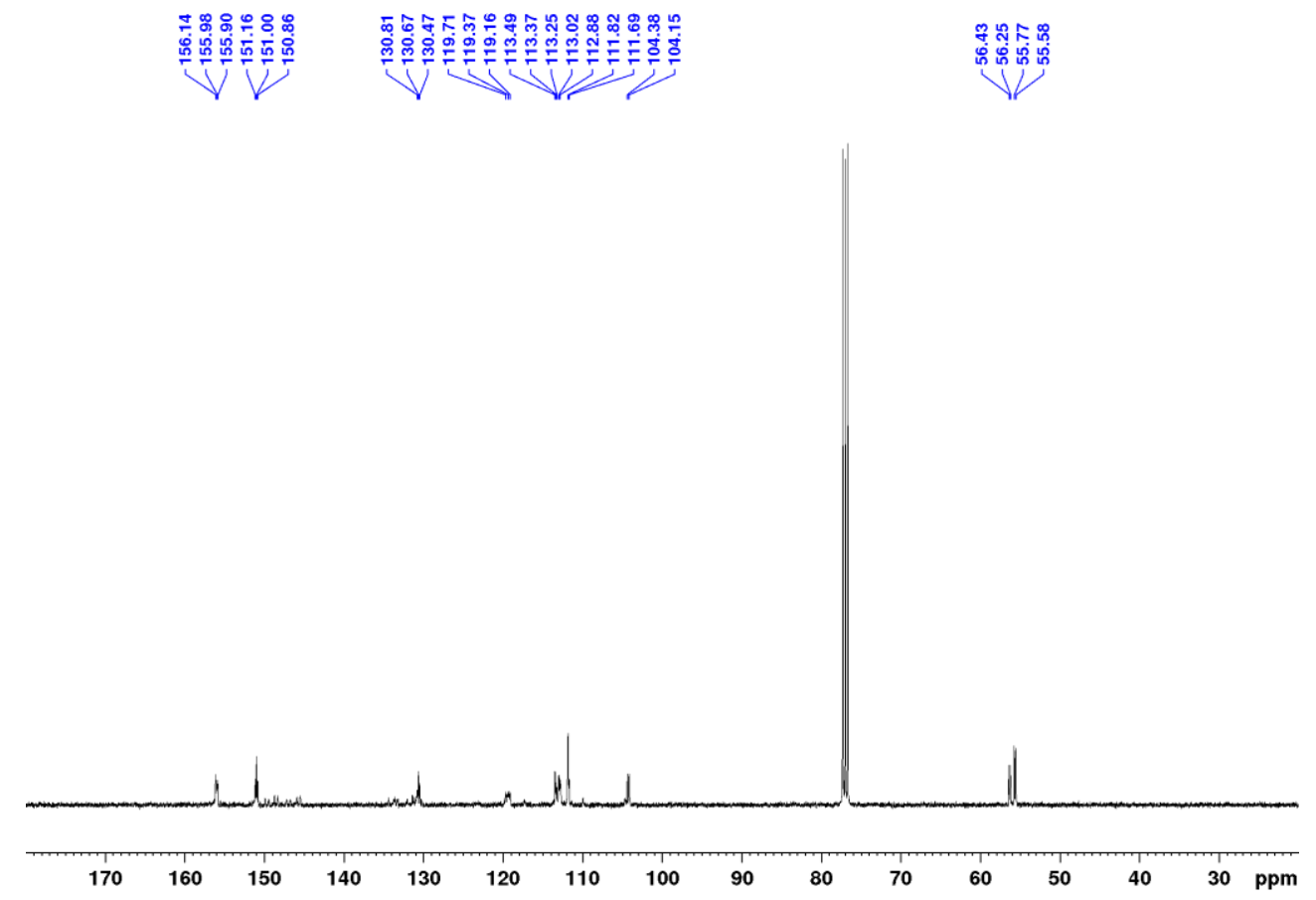


TGA

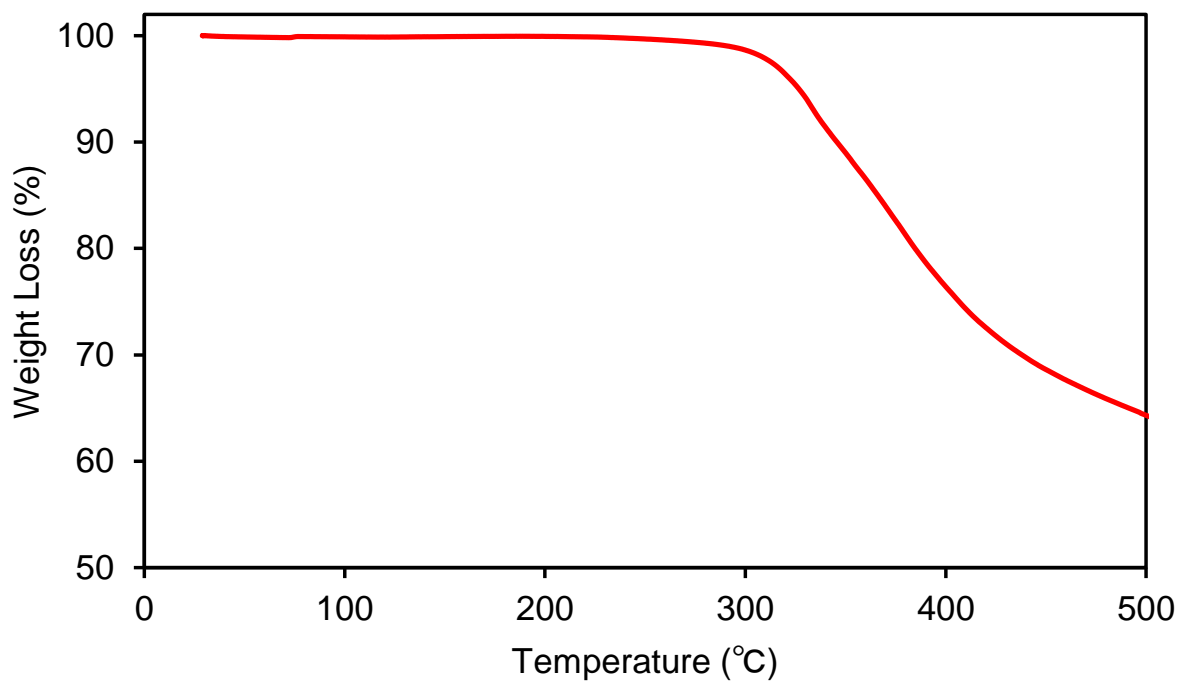

DSC

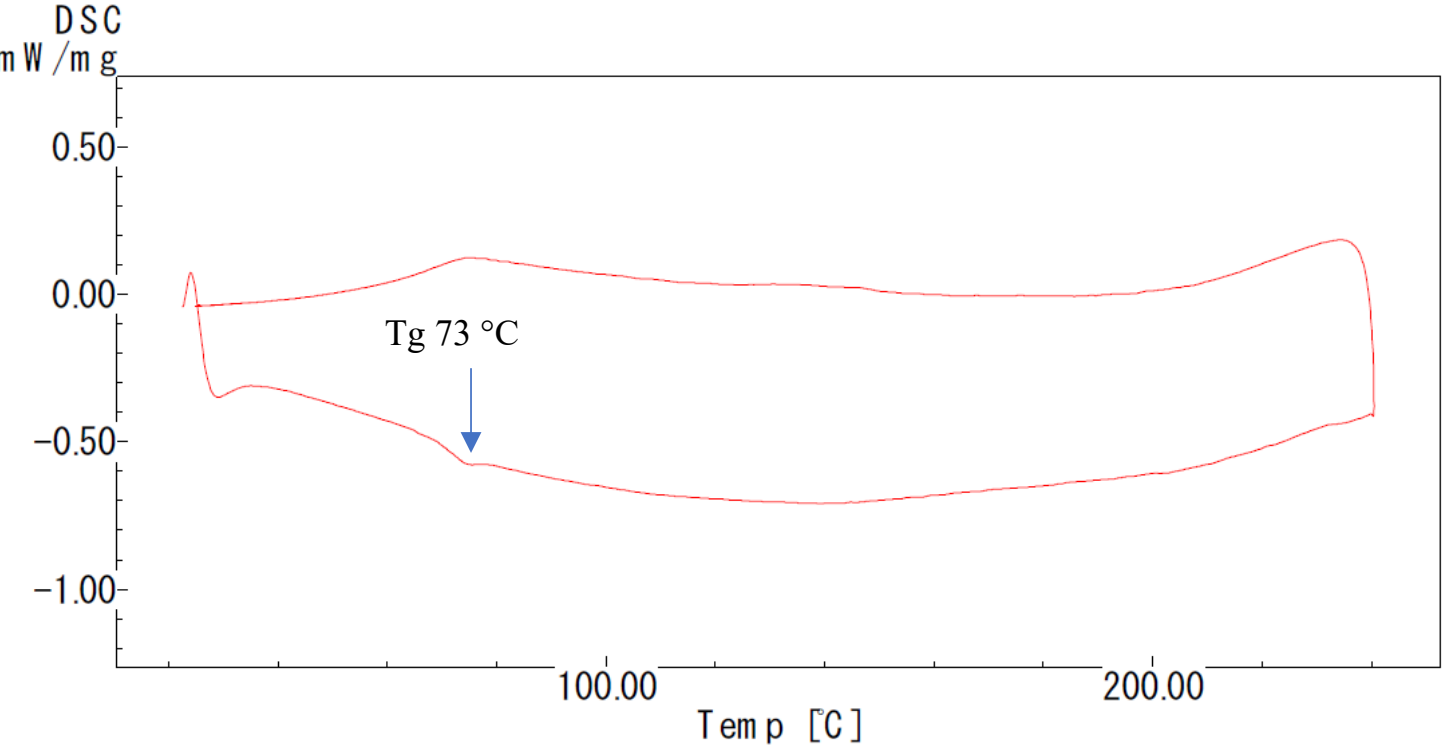


UV/vis and PL spectra of the polymers

3b
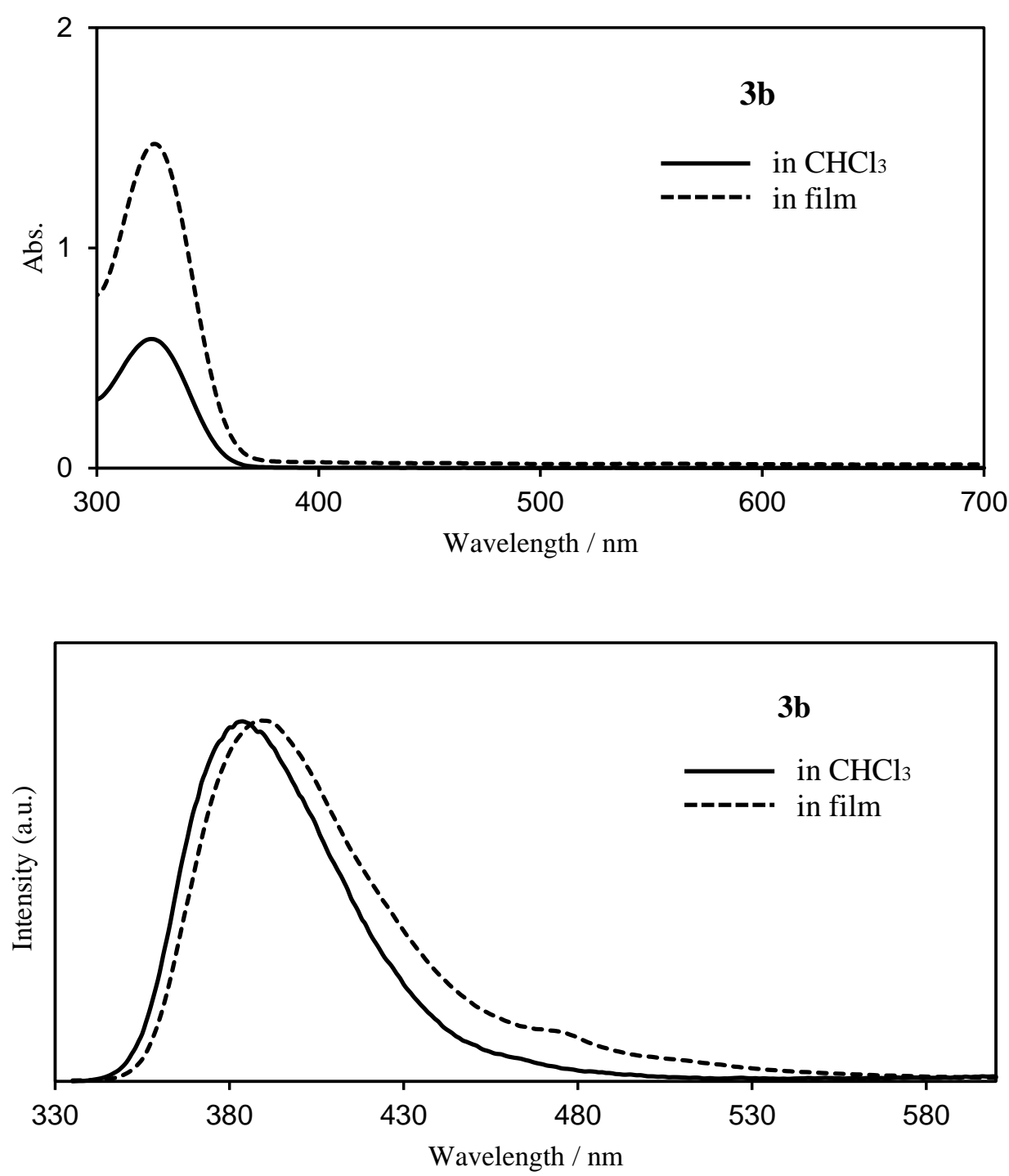

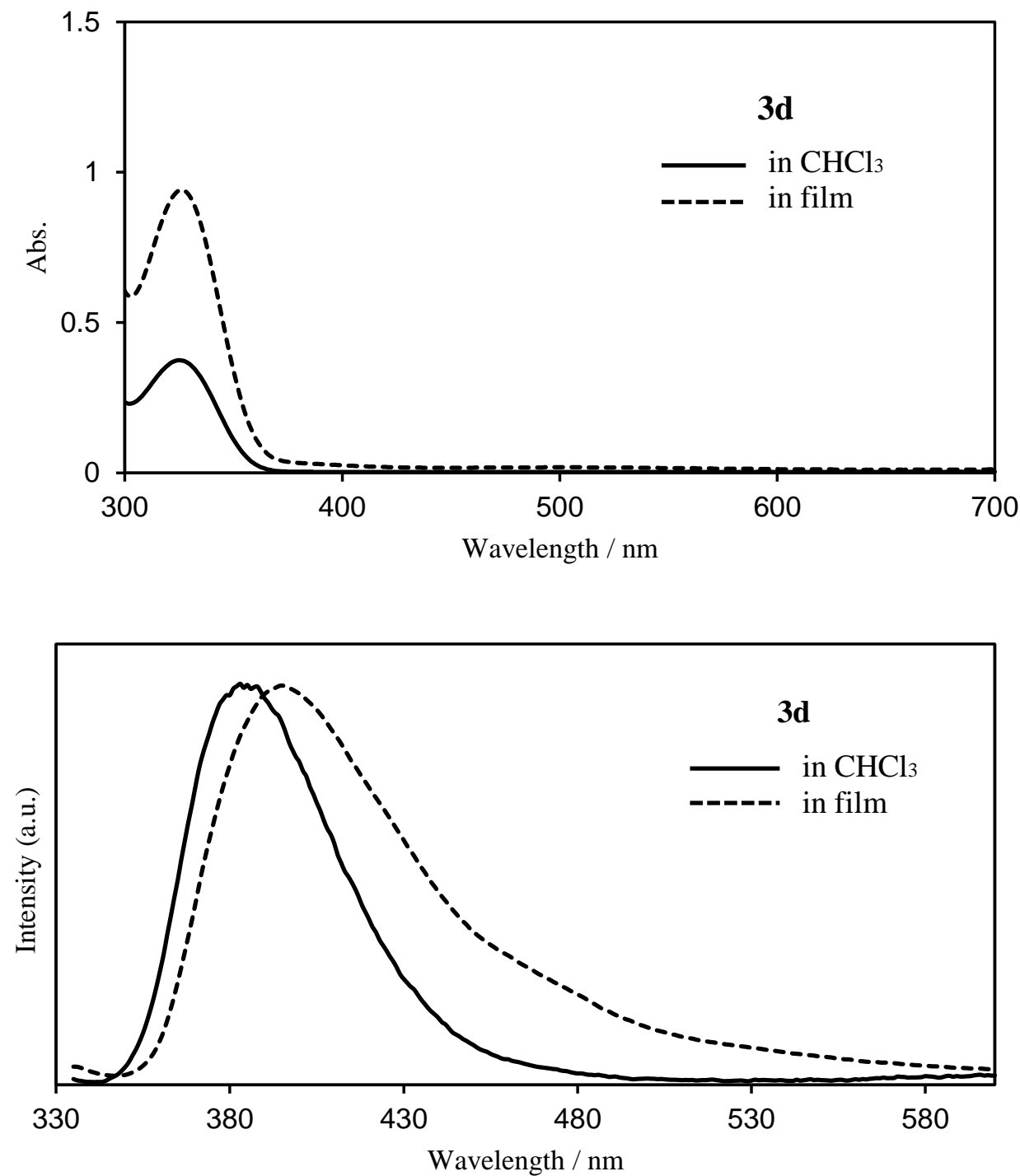

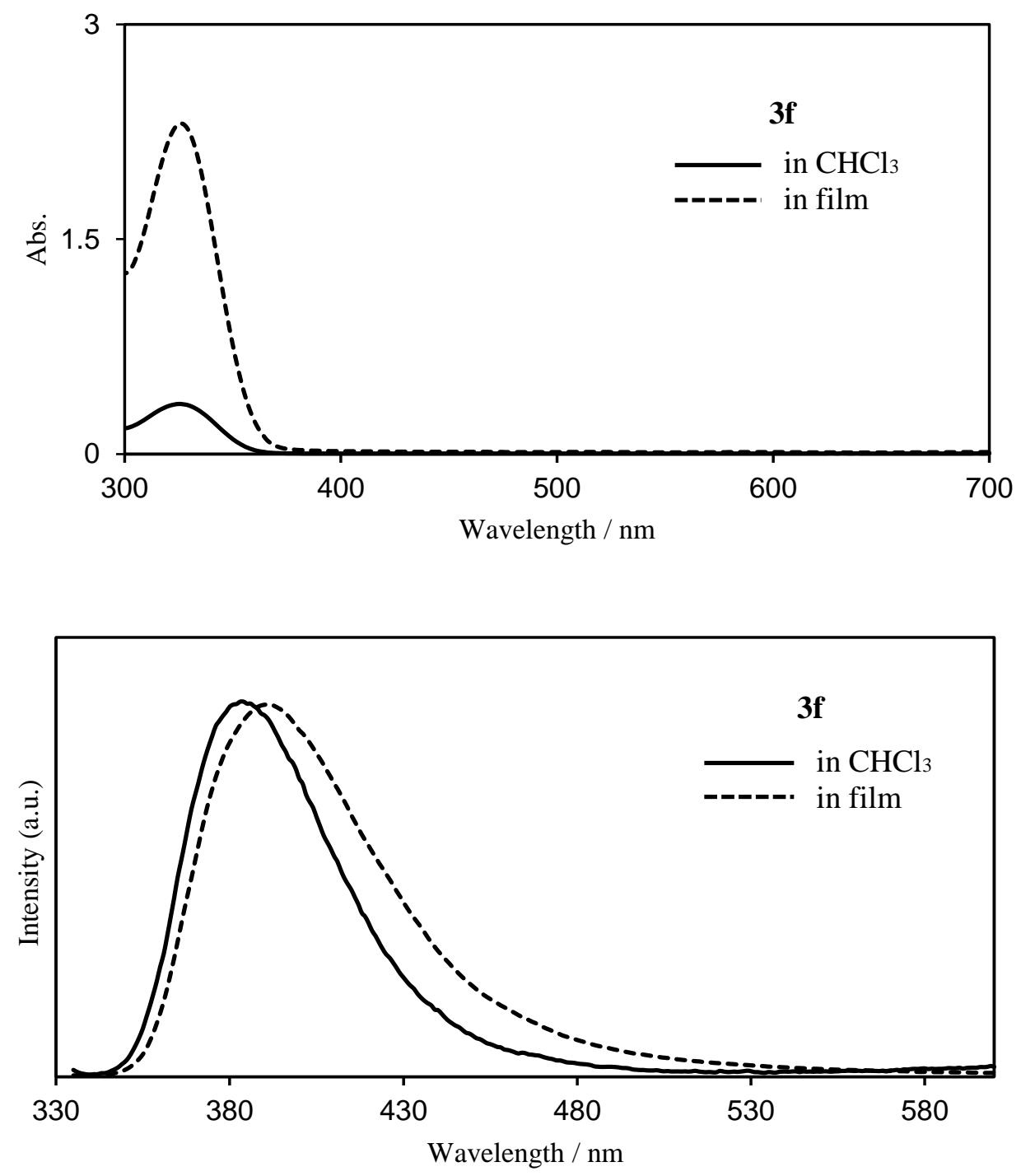
Contact angle measurement.

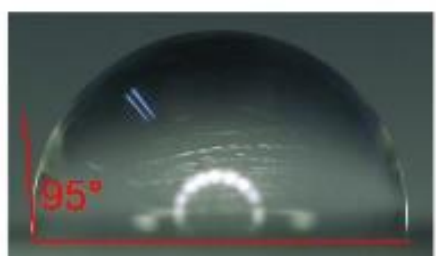

This photo-image shows the water droplet on the cast film of $\mathbf{3 c}$, which was prepared using the EtOAc solution of $\mathbf{3 c}$ and a PFA substrate. 
Complete citation for ref. 38

Gaussian 16, Revision C.01, Frisch, M. J.; Trucks, G. W.; Schlegel, H. B.; Scuseria, G. E.; Robb, M. A.; Cheeseman, J. R.; Scalmani, G.; Barone, V.; Petersson, G. A.; Nakatsuji, H.; Li, X.; Caricato, M.; Marenich, A. V.; Bloino, J.; Janesko, B. G.; Gomperts, R.; Mennucci, B.; Hratchian, H. P.; Ortiz, J. V.; Izmaylov, A. F.; Sonnenberg, J. L.; Williams-Young, D.; Ding, F.; Lipparini, F.; Egidi, F.; Goings, J.; Peng, B.; Petrone, A.; Henderson, T.; Ranasinghe, D.; Zakrzewski, V. G.; Gao, J.; Rega, N.; Zheng, G.; Liang, W.; Hada, M.; Ehara, M.; Toyota, K.; Fukuda, R.; Hasegawa, J.; Ishida, M.; Nakajima, T.; Honda, Y.; Kitao, O.; Nakai, H.; Vreven, T.; Throssell, K.; Montgomery, J. A., Jr.; Peralta, J. E.; Ogliaro, F.; Bearpark, M. J.; Heyd, J. J.; Brothers, E. N.; Kudin, K. N.; Staroverov, V. N.; Keith, T. A.; Kobayashi, R.; Normand, J.; Raghavachari, K.; Rendell, A. P.; Burant, J. C.; Iyengar, S. S.; Tomasi, J.; Cossi, M.; Millam, J. M.; Klene, M.; Adamo, C.; Cammi, R.; Ochterski, J. W.; Martin, R. L.; Morokuma, K.; Farkas, O.; Foresman, J. B.; Fox, D. J. Gaussian, Inc., Wallingford CT, 2019. 